\title{
Optimum Mode-Switching-Assisted Constant-Power Single- and Multicarrier Adaptive Modulation
}

\author{
Byoungjo Choi and Lajos Hanzo, Senior Member, IEEE
}

\begin{abstract}
A set of optimum mode-switching levels is derived for a generic constant-power adaptive-modulation scheme based on a closed-form expression of the average bit error ratio (BER) and the average bits-per-symbol (BPS) throughput of the adaptive-modulation scheme. This results in a constant BER, variable-throughput arrangement. The corresponding BPS throughput performance and the achievable signal-to-noise ratio (SNR) gain are investigated for the optimum mode-switching assisted constant-power adaptive-modulation schemes employing various diversity schemes, including maximal ratio combining (MRC) receive-antenna diversity, a two-dimensional RAKE receiver, as well as transmit-diversity aided space-time (ST) coding, when communicating over various fading scenarios. The BPS throughput of our constant-power adaptive quadrature amplitude modulation (AQAM) scheme approaches the throughput of variable-power variable-rate $A Q A M$ within $1 \mathrm{~dB}$. However, the achievable throughput gain of the adaptive-modulation scheme, in comparison to conventional fixed-mode modems, is substantially reduced as the diversity order of the receiver is increased. Hence, adaptive modulation constitutes a lower complexity alternative to multiple-transmitter and receiver-based systems when considering the range of techniques that can be used for mitigating the effects of the channel-quality fluctuations imposed by wireless channels.
\end{abstract}

Index Terms-Adaptive modulation, adaptive quadrature amplitude modulation (AQAM), fading counter measures, Lagrangian optimization for adaptive modulation, optimum switching levels for adaptive modulation.

\section{INTRODUCTION}

$\mathbf{M}$ OBILE COMMUNICATIONS channels typically exhibit time-variant channel-quality fluctuations [1] and [2] and, hence, conventional fixed-mode modems suffer from bursts of transmission errors, even if the system was designed to provide a high link margin. An efficient approach to mitigating these detrimental effects is to adaptively adjust the transmission format based on the near-instantaneous channel-quality information perceived by the receiver, which is fed back to the transmitter with the aid of a feedback channel [2]-[4]. This scheme requires a reliable feedback link from the receiver to the transmitter. The channel-quality variation should be sufficiently slow for the transmitter to be able to adapt. Hayes [3] proposed transmission-power adaptation, while Cavers [5] suggested invoking a variable symbol-duration scheme in response to the perceived channel quality at the expense of a variable bandwidth requirement. Since a variable-power scheme increases the peak level of cochannel interference [6]

Manuscript received October 17, 2001; revised May 31, 2002.

The authors are with the Department of Electronics and Computer Science,

University of Southampton SO17 1BJ, U.K. (e-mail: lh@ecs.soton.ac.uk).

Digital Object Identifier 10.1109/TVT.2003.810970 imposed on other users of the system, rather than variable bit rate but constant symbol rate, adaptive quadrature amplitude modulation (AQAM) was proposed by Steele and Webb as an alternative, while employing various star quadrature amplitude modulation (QAM) constellations [6] and [7]. With the advent of pilot symbol-assisted modulation (PSAM) [8]-[10], Otsuki et al. [11] employed maximum-minimum distance-based square constellations rather than star constellations in the context of AQAM, as a practical fading-counter measure. Analyzing the channel capacity of Rayleigh-fading channels [12]-[14], Goldsmith et al. showed that variable power, variable-rate adaptive schemes are optimum, approaching the capacity of the channel and characterizing the throughput performance of variable-power AQAM [13]. However, they also found that the extra throughput achieved by the additional variable-power-assisted adaptation over the constant-power variable-rate scheme is marginal for most types of fading channels [13] and [15].

Since the performance of the AQAM scheme is predetermined by the switching levels employed, various attempts have been made to determine the suitable switching levels. An attempt to find optimum switching levels that are capable of satisfying various transmission-integrity requirements was made by Webb and Steele [6]. They used the bit error ratio (BER) curves of each constituent-modulation mode, obtained from conducted simulations when communicating over an additive white Gaussian noise (AWGN) channel, in order to find the signal-to-noise ratio (SNR) values, where each modulation mode satisfies the target BER requirement [2]. This intuitive concept of determining switching levels has been widely used by researchers [11] and [15] since then. The regime proposed by Webb and Steele can be used for ensuring that instantaneous BER always remains below a certain threshold BER $P_{t h}$. However, no direct relationship between the resultant-average BER of the corresponding adaptive modem and the peak-instantaneous BER $P_{t h}$ was available.

In order to generate the optimum switching levels that satisfy the target average BER, while maximizing the obtainable bits-per-symbol (BPS) throughput, Torrance and Hanzo [16] proposed the employment of a heuristic cost function and applied Powell's optimization method [17]. The multidimensional optimization of the cost function produces a set of constant switching levels across the entire SNR range and the corresponding average BER exhibited good agreement with the desired target average BER. However, the actual average BER of the adaptive scheme was not constant across the SNR range, implying that the average throughput could potentially be increased further. Hence, a per SNR optimization scheme em- 


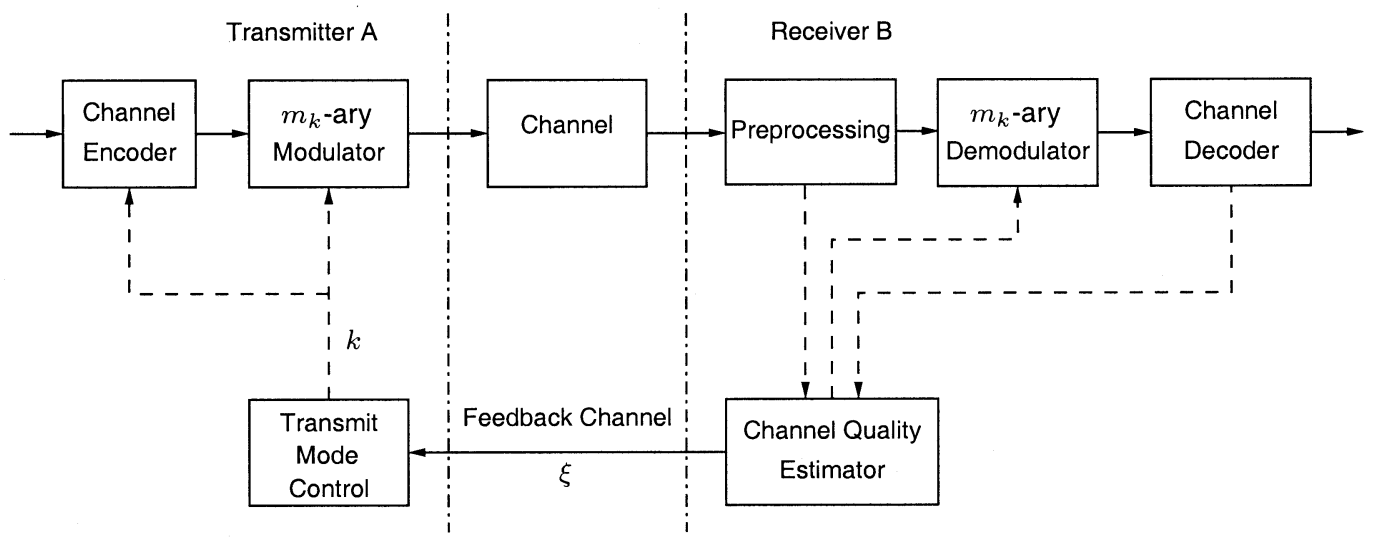

Fig. 1. Stylized model of the near-instantaneously adaptive-modulation scheme.

ploying a modified cost function was developed to guarantee a constant target average BER across the whole operating SNR range [18]. Since Powell's multidimensional optimization may be trapped in a local optimum, rather than identifying the global optimum, the previous two schemes often result in suboptimal switching levels. Recently, Paris et al. [19] proposed a set of optimal switching thresholds based on a multidimensional optimization technique and an approximated representation of the BER of the constituent QAM modes. Chung and Goldsmith [20] also provided a comprehensive set of formulas for determining the optimum switching thresholds for various types of AQAM schemes. In [20], a set of suboptimal thresholds were used for the constant-power AQAM scheme under the constraint of requiring a fixed-average BER.

In this contribution, we derive an exact closed-form expression for the average BER of our constant-power adaptive-modulation schemes employing various phase-shift keying (PSK)- and square-QAM constellations and then apply the Lagrangian optimization technique for deriving the globally optimized modulation-mode switching levels. The relationship between the optimum-mode switching levels is established, which holds regardless of the underlying channel scenario. Then the corresponding optimum-mode switching-assisted constant-power adaptive-modulation schemes are investigated in various operating scenarios, leading to plausible performance results and to our discussions.

The next section introduces a general model of the constantpower adaptive-modulation schemes considered and defines a set of parameters characterizing their performance, followed by the determination of their closed-form expressions when communicating over a narrow-band Nakagami-fading channel. Section III derives the optimum switching levels with the aid of the Lagrangian-based optimization technique. The performance results of our constant-power AQAM schemes employing various diversity schemes operating in various fading scenarios are presented in Section IV, followed by our conclusions in Section V.

\section{SySTEM DESCRIPTION}

A stylized model of our adaptive-modulation scheme is illustrated in Fig. 1. In our adaptive-modulation scheme, the modulation mode used is adapted on a near-instantaneous basis for the sake of counteracting the effects of fading. Let us describe the detailed operation of the adaptive-modem scheme of Fig. 1. First, the channel quality $\xi$ is estimated by the remote receiver B of Fig. 1. This channel-quality measure $\xi$ can be the instantaneous channel SNR, the radio-signal strength indicator (RSSI) output of the receiver [6], the decoded BER [6], the signal-to-interference-and-noise ratio (SINR) estimated at the output of the channel equalizer [21], or the SINR at the output of a CDMA joint detector [22]. The estimated channel quality perceived by receiver $B$ is fed back to transmitter $A$ with the aid of a feedback channel, as seen in Fig. 1. Then, the transmit mode control block of transmitter A of Fig. 1 selects the highest throughput modulation mode $k$ capable of maintaining the target BER based on the channel-quality measure $\xi$ and the specific set of adaptive mode-switching levels s. Once $k$ is selected, $m_{k}$-ary modulation is performed at transmitter A in order to generate the transmitted signal $s(t)$ and the signal $s(t)$ is transmitted through the channel.

\section{A. Modulation Mode-Switching Model}

A $K$-mode adaptive-modulation scheme adjusts its transmit mode $k$, where $k \in\{0,1 \ldots K-1\}$, by employing $m_{k}$-ary modulation according to the near-instantaneous channel quality $\xi$ perceived by receiver B of Fig. 1 . The mode selection rule is given by

$$
\text { Choose mode } k \text { when } s_{k} \leq \xi<s_{k+1}
$$

where a switching level $s_{k}$ belongs to the set $\mathbf{s}=\left\{s_{k} \mid k=\right.$ $0,1, \ldots, K\}$. The BPS throughput $b_{k}$ of a specific modulation mode $k$ is given by $b_{k}=\log _{2}\left(m_{k}\right)$ if $m_{k} \neq 0$, otherwise $b_{k}=0$. It is convenient to define the incremental BPS $c_{k}$ as $c_{k}=b_{k}-b_{k-1}$ when $k>0$ and $c_{0}=b_{0}$, which quantifies the achievable BPS increase when switching from the lower throughput mode $k-1$ to mode $k$.

\section{B. Example: Five-Mode AQAM}

A five-mode AQAM system has been studied extensively by numerous researchers, which was motivated by the high performance of the Gray-mapped constituent modulation modes used. The parameters of this five-mode AQAM system are summarized in Table I. In our investigations, the near-instantaneous channel quality $\xi$ is defined as the instantaneous channel SNR $\gamma$. The boundary-switching levels are given as $s_{0}=0$ and $s_{5}=\infty$. 


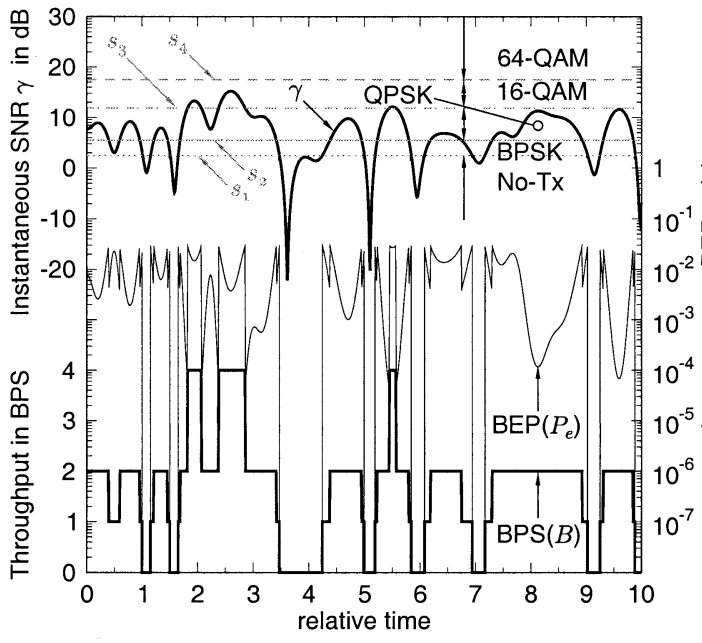

(a)

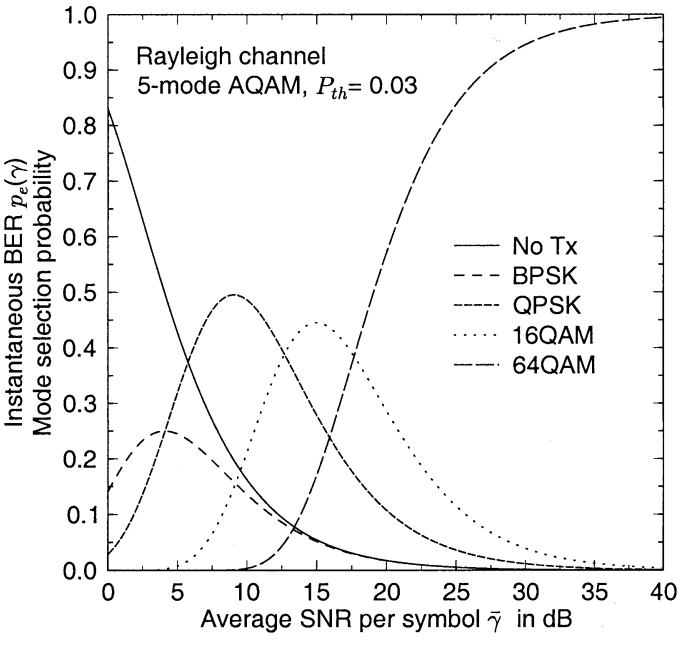

(b)

Fig. 2. Various characteristics of the five-mode AQAM scheme communicating over a Rayleigh-fading channel employing the specific set of switching levels designed for limiting the peak instantaneous BER to $P_{t h}=3 \times 10^{-2}$. (a) The evolution of the instantaneous channel SNR $\gamma$ is represented by the thick line at the top of the graph, the associated instantaneous BER $p_{e}(\gamma)$ by the thin line in the middle and the instantaneous BPS throughput $b(\gamma)$ by the thick line at the bottom. The average SNR is $\bar{\gamma}=10 \mathrm{~dB}$. (b) As the average SNR increases, the higher order AQAM modes are selected more often.

TABLE I

THE PARAMETERS OF FIVE-MODE AQAM SYSTEM

\begin{tabular}{c||c|c|c|c|c}
\hline \hline$k$ & 0 & 1 & 2 & 3 & 4 \\
\hline$m_{k}$ & 0 & 2 & 4 & 16 & 64 \\
\hline$b_{k}$ & 0 & 1 & 2 & 4 & 6 \\
\hline$c_{k}$ & 0 & 1 & 1 & 2 & 2 \\
\hline mode & No Tx & BPSK & QPSK & 16-QAM & 64-QAM \\
\hline \hline
\end{tabular}

Fig. 2(a) illustrates the operation of the five-mode AQAM scheme when communicating over a typical narrow-band Rayleigh-fading channel scenario. Transmitter A of Fig. 1 keeps track of the channel SNR $\gamma$ perceived by receiver B with the aid of a low BER low-delay feedback channel-which can be created, for example, by superimposing the values of $\xi$ on the reverse-direction transmitted messages of transmitter $\mathrm{B}$ - and determines the highest BPS modulation mode maintaining the target BER, depending on which region $\gamma$ falls into. The channel-quality related SNR regions are divided by the modulation mode switching levels $s_{k}$. The peak instantaneous BER (iBER) limiting scheme [6] was used for deriving the switching levels used in Fig. 2(a). We assumed the peak iBER of $P_{t h}=3 \times 10^{-2}$ in Fig. 2(a). The associated instantaneous BPS throughput $b$ is also depicted, using the thick stepped line at the bottom of Fig. 2(a). We can observe that the throughput varied from 0 BPS, when the no-transmission (No-Tx) AQAM mode was chosen, to 4 BPS, when the 16-QAM mode was activated. During the depicted observation window, the 64-QAM mode was not activated. The instantaneous BER, depicted as a thin line using the middle trace of Fig. 2(a), is limited by the peak iBER of $P_{t h}=3 \times 10^{-2}$.

\section{Characteristic Parameters}

In this section, we introduce several parameters in order to characterize our adaptive-modulation scheme. The constituent mode selection probability (MSP) $\mathcal{M}_{k}$ is defined as the probability of selecting the $k$ th mode from the set of $K$ possible modulation modes, which can be calculated as a function of the channel-quality metric $\xi$, regardless of the specific metric used, as

$$
\begin{aligned}
\mathcal{M}_{k} & =\operatorname{Pr}\left[s_{k} \leq \xi<s_{k+1}\right] \\
& =\int_{s_{k}}^{s_{k+1}} f(\xi) d \xi
\end{aligned}
$$

where $s_{k}$ denotes the mode-switching levels and $f(\xi)$ is the probability density function (pdf) of $\xi$. Then, the average throughput $B$ expressed in terms of BPS can be described as

$$
\begin{aligned}
B & =\sum_{k=0}^{K-1} b_{k} \int_{s_{k}}^{s_{k+1}} f(\xi) d \xi \\
& =\sum_{k=0}^{K-1} b_{k} \mathcal{M}_{k}
\end{aligned}
$$

which in simple verbal terms can be formulated as the weighted sum of the throughput $b_{k}$ of the individual AQAM constituent modes, where the weighting takes into account the probability $\mathcal{M}_{k}$ of activating the various constituent modes. When $s_{K}=$ $\infty$, the average throughput $B$ can also be formulated as

$$
\begin{aligned}
B & =\sum_{k=0}^{K-1} b_{k} \int_{s_{k}}^{s_{k+1}} f(\xi) d \xi \\
& =\sum_{k=0}^{K-1}\left[b_{k} \int_{s_{k}}^{\infty} f(\xi) d \xi-b_{k} \int_{s_{k+1}}^{\infty} f(\xi) d \xi\right] \\
& =b_{0} \int_{s_{0}}^{\infty} f(\xi) d \xi+\sum_{k=1}^{K-1}\left(b_{k}-b_{k-1}\right) \int_{s_{k}}^{\infty} f(\xi) d \xi \\
& =\sum_{k=0}^{K-1} c_{k} \int_{s_{k}}^{\infty} f(\xi) d \xi=\sum_{k=0}^{K-1} c_{k} F_{c}\left(s_{k}\right)
\end{aligned}
$$


where $c_{k} \triangleq b_{k}-b_{k-1}$ and $F_{c}(\xi)$ is the complementary cumulative distribution function (cdf) defined as

$$
F_{c}(\xi) \triangleq \int_{\xi}^{\infty} f(x) d x
$$

Let us now assume that we use the instantaneous SNR $\gamma$ as the channel quality measure $\xi$, which implies that no cochannel interference is present. By contrast, when operating in a cochannel interference-limited environment, we can use the instantaneous SINR as the channel quality measure $\xi$, provided that the cochannel interference has a near-Gaussian distribution. In this scenario, the mode-specific average BER $P_{k}$ can be written as

$$
P_{k}=\int_{s_{k}}^{s_{k+1}} p_{m_{k}}(\gamma) f(\gamma) d \gamma
$$

where $p_{m_{k}}(\gamma)$ is the BER of the $m_{k}$-ary AQAM constituentmodulation mode for transmission over the AWGN channel. We used $\gamma$ rather than $\xi$ in order to explicitly indicate the employment of $\gamma$ as the channel quality measure. Then, the average BER $P_{\text {avg }}$ of our adaptive-modulation scheme can be represented as the sum of the BERs of the specific constituent modes divided by the average adaptive modem throughput $B$, formulated as [23]

$$
P_{\mathrm{avg}}=\frac{1}{B} \sum_{k=0}^{K-1} b_{k} P_{k}
$$

where $b_{k}$ is the BPS throughput of the $k$ th modulation mode, $P_{k}$ is the mode-specific average BER given in (10), and $B$ is the average adaptive modem throughput given in (5) or in (8).

The aim of our adaptive system is to transmit as high a number of bits per symbol as possible, while providing the required quality of service (QoS). More specifically, we are aiming to maximize the average BPS throughput $B$ of (4), while satisfying the average BER requirement of $P_{\text {avg }} \leq P_{t h}$. Hence, we have to satisfy the constraint of meeting $P_{t h}$ while optimizing the design parameter of $\mathbf{s}$, which is the set of modulation-mode switching levels. The determination of the optimum switching levels will be investigated in Section III. Since the calculation of the optimum switching levels typically requires the numerical computation of the parameters introduced in this section, it is advantageous to express the parameters in a closed form, which is the objective of the next section.

1) Closed-Form Expressions for Transmission Over Nakagami-Fading Channels: Fading channels are often modeled as Nakagami-fading channels [24]. The pdf of the instantaneous channel SNR $\gamma$ when communicating over a Nakagami-fading channel is given as [24]

$$
f(\gamma)=\left(\frac{m}{\bar{\gamma}}\right)^{m} \frac{\gamma^{m-1}}{\Gamma(m)} e^{-m \gamma / \bar{\gamma}}, \gamma \geq 0
$$

where the parameter $m$ governs the severity of the fading and $\Gamma(m)$ is the Gamma function [25]. When $m=1$, the pdf of (12) is reduced to the pdf of $\gamma$ over Rayleigh-fading channel. As $m$ increases, the fading behaves like Rician fading; it becomes the AWGN channel when $m$ tends to $\infty$. Here, we restrict the value of $m$ to be a positive integer. In this case, the Nakagami fading model of (12), having a mean of $\bar{\gamma}_{s}=m \bar{\gamma}$, will be used to describe the pdf of the SNR per symbol $\gamma_{s}$ in an $m$-antenna-based diversity-assisted system employing maximal ratio combining (MRC).

When the instantaneous channel SNR $\gamma$ is used as the channel quality measure $\xi$ in our adaptive-modulation scheme transmitting over a Nakagami channel, the parameters defined in Section II-C can be expressed in a closed form. Specifically, the MPS $\mathcal{M}_{k}$ can be expressed as

$$
\begin{aligned}
\mathcal{M}_{k} & =\int_{s_{k}}^{s_{k+1}} f(\gamma) d \gamma \\
& =F_{c}\left(s_{k}\right)-F_{c}\left(s_{k+1}\right)
\end{aligned}
$$

where the complementary $\operatorname{cdf} F_{c}(\gamma)$ is given by

$$
\begin{aligned}
F_{c}(\gamma) & =\int_{\gamma}^{\infty} f(x) d x \\
& =\int_{\gamma}^{\infty}\left(\frac{m}{\bar{x}}\right)^{m} \frac{x^{m-1}}{\Gamma(m)} e^{-m x / \bar{\gamma}} d x \\
& =e^{-m \gamma / \bar{\gamma}} \sum_{i=0}^{m-1} \frac{\left(\frac{m \gamma}{\bar{\gamma}}\right)^{i}}{\Gamma(i+1)} .
\end{aligned}
$$

In deriving (17), we used the result of the indefinite integral of [26]

$$
\left.\int x^{n} e^{-a x} d x=-\left(\frac{e^{-a x}}{a}\right) \sum_{i=0}^{n} \frac{x^{n-i}}{a^{i}} \frac{n !}{(n-i) !}\right) .
$$

In a Rayleigh fading scenario, i.e., when $m=1$, the MPS $\mathcal{M}_{k}$ of (14) can be expressed as

$$
\mathcal{M}_{k}=e^{-s_{k} / \bar{\gamma}}-e^{-s_{k+1} / \bar{\gamma}} .
$$

The average throughput $B$ of our adaptive-modulation scheme transmitting over a Nakagami channel is given by substituting (17) into (8), yielding

$$
B=\sum_{k=0}^{K-1} c_{k} e^{-m s_{k} / \bar{\gamma}}\left\{\sum_{i=0}^{m-1} \frac{\left(\frac{m s_{k}}{\bar{\gamma}}\right)^{i}}{\Gamma(i+1)}\right\} .
$$

Let us now derive the closed-form expressions for the modespecific average BER $P_{k}$, defined in (10) for the various modulation modes when communicating over a Nakagami channel. The BER of a Gray-coded square QAM constellation for transmission over AWGN channels is given as [2]

$$
p_{m_{k}, \mathrm{QAM}}(\gamma)=\sum_{i} A_{i} Q\left(\sqrt{a_{i} \gamma}\right)
$$

where the values of the constants $A_{i}$ and $a_{i}$ were given in (47) of Appendix A. Then, the mode-specific average BER $P_{k}, \mathrm{QAM}$ of $m_{k}$-ary QAM over a Nakagami channel can be expressed as in 
Appendix B, yielding (see (22)-(24) at the bottom of the page) where $g(\gamma)]_{s_{k}}^{s_{k+1}} \triangleq g\left(s_{k+1}\right)-g\left(s_{k}\right)$ and $X_{j}$ is given by

$$
\begin{aligned}
X_{j}\left(\gamma, a_{i}\right)= & \frac{\mu^{2}}{\sqrt{2 a_{i} \pi}}\left(\frac{m}{\bar{\gamma}}\right)^{j} \frac{\Gamma\left(j+\frac{1}{2}\right)}{\Gamma(j+1)} \\
& \times \sum_{k=1}^{j}\left(\frac{2 \mu^{2}}{a_{i}}\right)^{j-k} \frac{\gamma^{k-1 / 2}}{\Gamma\left(k+\frac{1}{2}\right)} e^{-a_{i} \gamma /\left(2 \mu^{2}\right)} \\
& +\left(\frac{2 \mu^{2} m}{a_{i} \bar{\gamma}}\right)^{j} \frac{1}{\sqrt{\pi}} \frac{\Gamma\left(j+\frac{1}{2}\right)}{\Gamma(j+1)} \mu Q\left(\frac{\sqrt{a_{i} \gamma}}{\mu}\right)
\end{aligned}
$$

where, again, $\mu \triangleq \sqrt{a_{i} \bar{\gamma} /\left(2+a_{i} \bar{\gamma}\right)}$ and $\Gamma(x)$ is the Gamma function.

On the other hand, the high-accuracy approximated BER formula of a Gray-coded $m_{k}$-ary PSK scheme $(k \geq 3)$ transmitting over an AWGN channel is given as [27]

$$
\begin{aligned}
p_{m_{k}, \mathrm{PSK}} & \simeq \frac{2}{k}\left\{Q\left(\sqrt{2 \gamma} \sin \left(\frac{\pi}{2^{k}}\right)\right)+Q\left(\sqrt{2 \gamma} \sin \left(\frac{3 \pi}{2^{k}}\right)\right)\right\} \\
& =\sum_{i} A_{i} Q\left(\sqrt{a_{i} \gamma}\right)
\end{aligned}
$$

where the set of constants $\left\{\left(A_{i}, a_{i}\right)\right\}$ is given by $\left\{\left(2 / k, 2 \sin ^{2}\left(\pi / m_{k}\right)\right),\left(2 / k, 2 \sin ^{2}\left(3 \pi / m_{k}\right)\right)\right\}$. The same expression as (21) and (27) can be applied to various coherent star QAM schemes [28]. Hence, the mode-specific average BER $P_{k, \text { PSK }}$ employing PSK constellations and the corresponding BER of $P_{k \text {,SQAM }}$ when employing star QAM constellations can be represented using the same equation, namely (24), as for $P_{k, \mathrm{QAM}}$.

\section{OPTIMUM SWITCHING LEVELS}

In this section, we restrict our interest to adaptive-modulation schemes employing the SNR per symbol $\gamma$ as the channel quality measure $\xi$. Our aim is to optimize the set of switching levels s such that the average BPS throughput $B(\bar{\gamma} ; \mathbf{s})$ can be maximized under the constraint of $P_{\text {avg }}(\bar{\gamma} ; \mathbf{s})=P_{t h}$.

Let us define $P_{R}$ for a $K$-mode adaptive-modulation scheme as the sum of the mode-specific average BER weighted by the BPS throughput of the individual constituent mode

$$
P_{R}(\bar{\gamma} ; \mathbf{s}) \triangleq \sum_{k=0}^{K-1} b_{k} P_{k}
$$

where $\bar{\gamma}$ is the average SNR per symbol, $\mathbf{s}$ is the set of switching levels, $K$ is the number of constituent modulation modes, $b_{k}$ is the BPS throughput of the $k$ th constituent mode, and the mode-specific average BER $P_{k}$ is given in (10). Explicitly, (10) implies weighting the BER $p_{m_{k}}(\gamma)$ by its probability of occurrence quantified in terms of its pdf and then averaging, i.e., integrating it over the range spanning from $s_{k}$ to $s_{k+1}$. Then, with the aid of (11), the average BER constraint can also be written as

$$
P_{\mathrm{avg}}(\bar{\gamma} ; \mathbf{s})=P_{t h} \Longleftrightarrow P_{R}(\bar{\gamma} ; \mathbf{s})=P_{t h} B(\bar{\gamma} ; \mathbf{s}) .
$$

Another logical constraint regarding the switching levels can be expressed as

$$
s_{k} \leq s_{k+1} .
$$

As we discussed before, our optimization goal is to maximize the objective function $B(\bar{\gamma} ; \mathbf{s})$ under the constraint of (29). The set of switching levels $\mathbf{s}$ has $K+1$ levels in it. However, considering that often we have $s_{0}=0$ and $s_{K}=\infty$ in various adaptive-modulation schemes, we have $K-1$ independent variables in $\mathbf{s}$. Hence, the optimization task is a $K-1$ dimensional optimization under a constraint [29]. It is a standard practice to introduce a modified objective function using a Lagrangian multiplier and to convert the problem into a set of one-dimensional (1-D) optimization problems. The modified objective function $\Lambda$ can be formulated employing a Lagrangian multiplier $\lambda$ [29] as

$$
\begin{aligned}
\Lambda(\mathbf{s} ; \bar{\gamma}) & =B(\bar{\gamma} ; \mathbf{s})+\lambda\left\{P_{R}(\bar{\gamma} ; \mathbf{s})-P_{t h} B(\bar{\gamma} ; \mathbf{s})\right\} \\
& =\left(1-\lambda P_{t h}\right) B(\bar{\gamma} ; \mathbf{s})+\lambda P_{R}(\bar{\gamma} ; \mathbf{s}) .
\end{aligned}
$$

The optimum set of switching levels should satisfy

$$
\begin{aligned}
\frac{\partial \Lambda}{\partial \mathbf{s}}=\frac{\partial}{\partial \mathbf{s}}\left(B(\bar{\gamma} ; \mathbf{s})+\lambda\left\{P_{R}(\bar{\gamma} ; \mathbf{s})-P_{t h} B(\bar{\gamma} ; \mathbf{s})\right\}\right) & =0 \\
\text { and } P_{R}(\bar{\gamma} ; \mathbf{s})-P_{t h} B(\bar{\gamma} ; \mathbf{s}) & =0 .
\end{aligned}
$$

By expanding (33) and eliminating the Lagrangian $\lambda$ as shown in Appendix C, we arrive at the following set of relationships between the optimum switching levels:

$$
y_{k}\left(s_{k}\right)=y_{1}\left(s_{1}\right) \text { for } k=2,3, \ldots K-1
$$

$$
\begin{aligned}
P_{k, \mathrm{QAM}} & =\int_{s_{k}}^{s_{k+1}} p_{m_{k}, \mathrm{QAM}}(\gamma) f(\gamma) d \gamma \\
& =\sum_{i} A_{i} \int_{s_{k}}^{s_{k+1}} Q\left(\sqrt{a_{i} \gamma}\right)\left(\frac{m}{\bar{\gamma}}\right)^{m} \frac{\gamma^{m-1}}{\Gamma(m)} e^{-m \gamma / \bar{\gamma}} d \gamma \\
& \left.\left.=\sum_{i} A_{i}\left\{-e^{-m \gamma / \bar{\gamma}} Q\left(\sqrt{a_{i} \gamma}\right) \sum_{j=0}^{m-1} \frac{\left(\frac{m \gamma}{\bar{\gamma}}\right)^{j}}{\Gamma(j+1)}\right]_{s_{k}}^{s_{k+1}}+\sum_{j=0}^{m-1} X_{j}\left(\gamma, a_{i}\right)\right]_{s_{k}}^{s_{k+1}}\right\}
\end{aligned}
$$




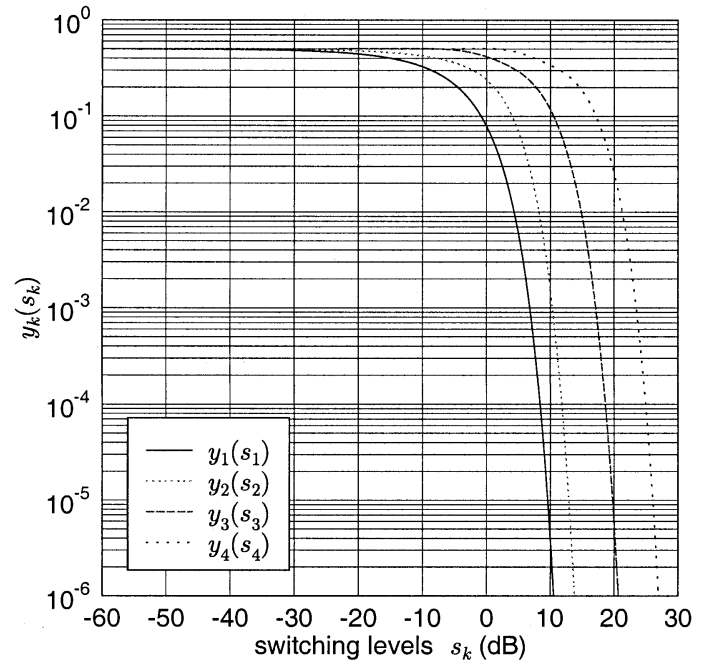

(a)

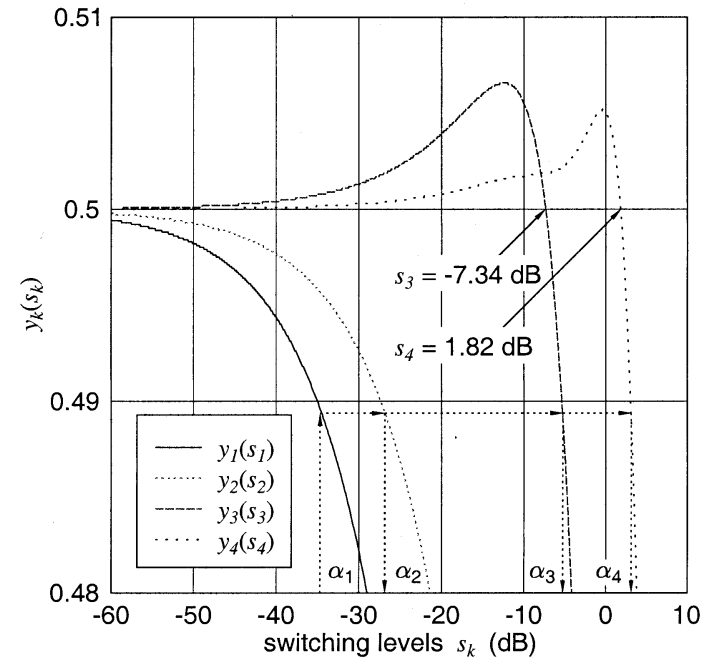

(b)

Fig. 3. The Lagrangian-free functions $y_{k}\left(s_{k}\right)$ of (37) to (40) for Gray-mapped square-shaped QAM constellations. As $s_{k}$ becomes lower, $y_{k}\left(s_{k}\right)$ asymptotically approaches 0.5 . Observe that while $y_{1}\left(s_{1}\right)$ and $y_{2}\left(s_{2}\right)$ are monotonic functions, $y_{3}\left(s_{3}\right)$ and $y_{4}\left(s_{4}\right)$ cross the $y=0.5$ line.

where the function $y_{k}\left(s_{k}\right)$ is defined as

$$
\begin{array}{r}
y_{k}\left(s_{k}\right) \triangleq \frac{1}{c_{k}}\left\{b_{k} p_{m_{k}}\left(s_{k}\right)-b_{k-1} p_{m_{k-1}}\left(s_{k}\right)\right\} \\
k=2,3, \ldots K-1
\end{array}
$$

which does not contain the Lagrangian multiplier $\lambda$ and, hence, will be referred to as the "Lagrangian-free function."

This function can be physically interpreted as the normalized BER difference between the adjacent adaptive-modulation modes. For example, $y_{1}\left(s_{1}\right)=p_{2}\left(s_{1}\right)$ quantifies the BER increase when switching from the No-Tx mode to the binary phase-shift keying (BPSK) mode, while $y_{2}\left(s_{2}\right)=2 p_{4}\left(s_{2}\right)-p_{2}\left(s_{2}\right)$ indicates the BER difference between the QPSK and BPSK modes. These curve will be more explicitly discussed in the context of Fig. 3. The significance of (35) is that the relationship between the optimum switching levels $s_{k}$, where $k=2,3, \ldots K-1$, and the lowest optimum switching level $s_{1}$ is independent of the underlying propagation scenario. Only the constituent adaptive-modulation mode related parameters, such as $b_{k}, c_{k}$, and $p_{m_{k}}(\gamma)$ govern this relationship.

As an example, let us consider the five-mode AQAM scheme introduced in Section II-B. The parameters of the five-mode AQAM scheme are summarized in Table I. Substituting these parameters into (35) and (36), we have the following:

$$
\begin{aligned}
& y_{1}\left(s_{1}\right)=p_{2}\left(s_{1}\right) \\
& y_{2}\left(s_{2}\right)=2 p_{4}\left(s_{2}\right)-p_{2}\left(s_{2}\right) \\
& y_{3}\left(s_{3}\right)=2 p_{16}\left(s_{3}\right)-p_{4}\left(s_{3}\right) \\
& y_{4}\left(s_{4}\right)=3 p_{64}\left(s_{4}\right)-2 p_{16}\left(s_{4}\right) .
\end{aligned}
$$

The Lagrangian-free functions of (37) to (40) are depicted in Fig. 3 for Gray-mapped square-shaped QAM.

As these functions are basically linear combinations of BER curves associated with AWGN channels, they exhibit "waterfall- like" shapes and asymptotically approach 0.5 as the switching levels $s_{k}$ approach zero (or $-\infty$, expressed in decibels). While $y_{1}\left(s_{1}\right)$ and $y_{2}\left(s_{2}\right)$ are monotonic functions, $y_{3}\left(s_{3}\right)$ and $y_{4}\left(s_{4}\right)$ cross the $y=0.5$ line at $s_{3}=-7.34 \mathrm{~dB}$ and $s_{4}=1.82 \mathrm{~dB}$, respectively, as can be observed in Fig. 3(b). One should also notice that the trivial solutions of (35) are $y_{k}=0.5$ at $s_{k}=0$, $k=1,2,3,4$, as we have discussed in Appendix C.

For a given value of $s_{1}$, the other switching levels can be determined as $s_{2}=y_{2}^{-1}\left(y_{1}\left(s_{1}\right)\right), s_{3}=y_{3}^{-1}\left(y_{1}\left(s_{1}\right)\right)$ and $s_{4}=$ $y_{4}^{-1}\left(y_{1}\left(s_{1}\right)\right)$. Since deriving the analytical inverse function of $y_{k}$ is an arduous task, we can rely on a graphical or a numerical method. Fig. 3(b) illustrates an example of the graphical method. Specifically, when $s_{1}=\alpha_{1}$, we first find the point on the curve $y_{1}$ directly above the abscissa value of $\alpha_{1}$, and then draw a horizontal line across the corresponding point. From the crossover points found on the curves of $y_{2}, y_{3}$, and $y_{4}$, with the aid of the horizontal line, we can find the corresponding values of the other switching levels, namely those of $\alpha_{2}, \alpha_{3}$, and $\alpha_{4}$. In a numerical sense, this solution corresponds to a 1-D root-finding problem [17, Ch. 9]. Since the first Lagrangian-free equation is given as $y_{1}\left(s_{1}\right)=p_{m_{1}}\left(s_{1}\right)$ according to (36), $y_{1}\left(s_{1}\right)$ is monotonic and is restricted to the range of $1 / 2<y_{1}\left(s_{1}\right)<$ $\infty$. In general, however, $y_{k}\left(s_{k}\right)$ for $k>1$ may not be monotonic, potentially resulting in multiple roots that satisfy $y_{k}\left(s_{k}\right)=$ $y_{1}\left(s_{1}\right)$. Nevertheless, as we argued in Appendix C, we can establish a unique relationship between $s_{1}$ and $s_{k}$ without jeopardizing the optimality in our average SNR range of interest. The numerical results shown in Fig. 4 represent the direct relationship between the optimum switching level $s_{1}$ and the other optimum switching levels, namely $s_{2}, s_{3}$, and $s_{4}$. While the optimum value of $s_{2}$ shows a near-linear relationship with respect to $s_{1}$, those of $s_{3}$ and $s_{4}$ asymptotically approach two different constants, as $s_{1}$ becomes smaller. This corroborates the trends observed in Fig. 3(b), where $y_{3}\left(s_{3}\right)$ and $y_{4}\left(s_{4}\right)$ cross the $y=0.5$ line at $s_{3}=-7.34 \mathrm{~dB}$ and $s_{4}=1.82 \mathrm{~dB}$, respectively. Since the low-order modulation modes are abandoned at high average channel SNRs in order to increase the average throughput, the 


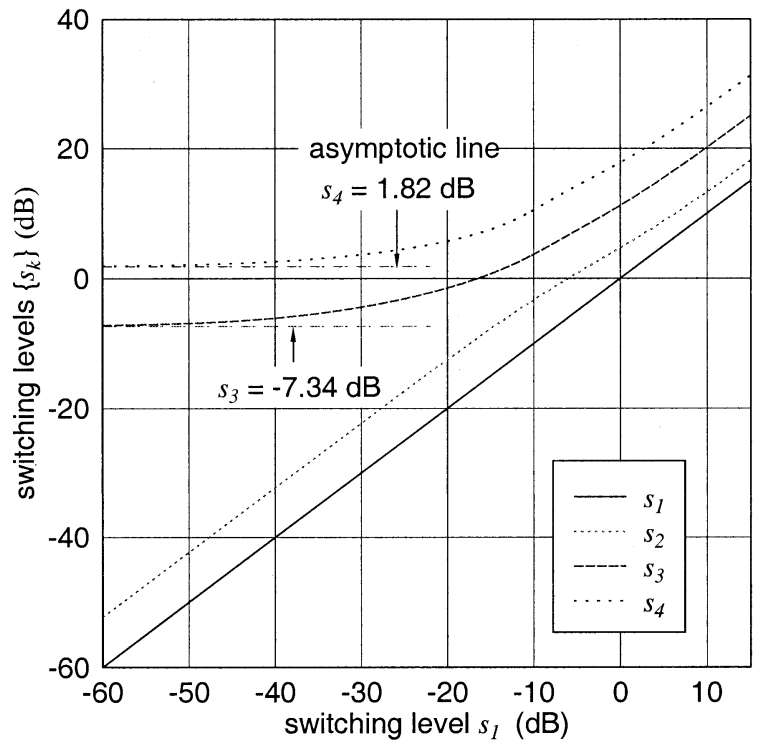

Fig. 4. Optimum switching levels as a function of $s_{1}$. Observe that while the optimum value of $s_{2}$ shows a linear relationship with respect to $s_{1}$, those of $s_{3}$ and $s_{4}$ asymptotically approach constant values as $s_{1}$ is reduced. A low value of $s_{1}$ corresponds to a high value of average SNR.

high values of $s_{1}$ on the horizontal axis of Fig. 4 indicate the encountering of a low-channel SNR, while low values of $s_{1}$ suggest that high-channel SNRs are experienced, as it transpires for example from Fig. 6.

Since we can relate the other switching levels to $s_{1}$, we have to determine the optimum value of $s_{1}$ for the given target BER, $P_{t h}$, and the pdf of the instantaneous channel SNR $f(\gamma)$ by solving the constraint equation given in (34). This problem also constitutes a 1-D root-finding problem, rather than a multidimensional optimization problem [16]-[18]. Let us define the constraint function $Y\left(\bar{\gamma} ; \mathbf{s}\left(s_{1}\right)\right)$ using (34) as

$$
Y\left(\bar{\gamma} ; \mathbf{s}\left(s_{1}\right)\right) \triangleq P_{R}\left(\bar{\gamma} ; \mathbf{s}\left(s_{1}\right)\right)-P_{t h} B\left(\bar{\gamma} ; \mathbf{s}\left(s_{1}\right)\right)
$$

where we represented the set of switching levels as a vector, which is the function of $s_{1}$, in order to emphasize that $s_{k}$ satisfies the relationships given by (35) and (36). More explicitly, $Y\left(\bar{\gamma} ; \mathbf{s}\left(s_{1}\right)\right)$ of (41) can be physically interpreted as the difference between $P_{R}\left(\bar{\gamma} ; \mathbf{s}\left(s_{1}\right)\right)$, namely the sum of the mode-specific average BERs weighted by the BPS throughput of the individual AQAM modes, as defined in (28), and the average BPS throughput $B\left(\bar{\gamma} ; \mathbf{s}\left(s_{1}\right)\right)$ weighted by the target BER $P_{t h}$. Considering the equivalence relationship given in (29), (41) reflects just another way of expressing the difference between the average BER $P_{\text {avg }}$ of the adaptive scheme and the target BER $P_{t h}$. Even though the relationships implied in $\mathbf{s}\left(s_{1}\right)$ are independent of the propagation conditions and the signaling power, the constraint function $Y\left(\bar{\gamma} ; \mathbf{s}\left(s_{1}\right)\right)$ of $(41)$ and, hence, the actual values of the optimum switching levels are dependent on the propagation conditions through the pdf $f(\gamma)$ of the SNR per symbol and on-the-average SNR per symbol $\bar{\gamma}$.

Let us find the initial value of $Y\left(\bar{\gamma} ; \mathbf{s}\left(s_{1}\right)\right)$ defined in (41), when $s_{1}=0$. An obvious solution for $s_{k}$ when $s_{1}=0$ is $s_{k}=0$ for $k=1,2, \ldots, K-1$. In this case, $Y\left(\bar{\gamma} ; \mathbf{s}\left(s_{1}\right)\right)$ becomes

$$
Y(\bar{\gamma} ; 0)=b_{K-1}\left(P_{m_{K-1}}(\bar{\gamma})-P_{t h}\right)
$$

where $b_{K-1}$ is the BPS throughput of the highest order constituent AQAM modulation mode, $P_{m_{K-1}}(\bar{\gamma})$ is the average BER of the highest order constituent modulation mode for transmission over the underlying channel scenario and $P_{t h}$ is the target average BER. The value of $Y(\bar{\gamma} ; 0)$ could be positive or negative, depending on the average SNR $\bar{\gamma}$ and on the target average BER $P_{t h}$. Let us denote the avalanche SNR by $\bar{\gamma}_{a}$ as such that we have $P_{m_{K-1}}\left(\bar{\gamma}_{a}\right)=P_{t h}$, where the highest order modulation mode satisfies the target average BER requirement. We observe that the modulation mode adaptation is abandoned when the average SNR becomes higher than the avalanche SNR, $\bar{\gamma}_{a}$. Another nonzero solution for $s_{k}$ may exist when $s_{1}=0$, if $y_{k}\left(s_{k}\right)$ crosses the horizontal line of $y_{k}=1 / 2$. This situation can be observed in Fig. 3(b) for $s_{3}$ and $s_{4}$. The corresponding value of $Y\left(\bar{\gamma} ; 0^{+}\right)$using this alternative solution turns out to be close to $Y(\bar{\gamma} ; 0)$. However, in the actual numerical evaluation of the initial value of $Y$, we should use $Y\left(\bar{\gamma} ; 0^{+}\right)$for ensuring the continuity of the function $Y$ at $s_{1}=0$.

Investigating the minima and the maxima of $Y$ as detailed in Appendix D, it was found that $Y\left(\bar{\gamma} ; s_{1}\right)$ has a global minimum of $Y_{\min }<0$ at $s_{1}=\zeta$ such that $b_{1} p_{m_{1}}(\zeta)-b_{0} p_{m_{0}}(\zeta)=$ $P_{t h}$ and a maximum of $Y_{\max 1}$ at $s_{1}=0$, as well as another maximum value $Y_{\max 2}=0^{-}$at $s_{1}=\infty$. This implies that there exists a unique set of optimum switching levels, when we have $Y_{\max 1}>0$ at $s_{1}=0$, i.e., when the average $\mathrm{SNR} \bar{\gamma}$ satisfies $\bar{\gamma}<\bar{\gamma}_{a}$.

As an example, let us evaluate the constraint function $Y\left(\bar{\gamma} ; s_{1}\right)$ for our five-mode AQAM scheme operating over a flat Rayleigh-fading channel. Fig. 5 depicts the values of $Y\left(s_{1}\right)$ for several values of the target average BER $P_{t h}$, when the average channel SNR is $30 \mathrm{~dB}$. We can observe that $Y\left(s_{1}\right)=0$ may have a root, depending on the target BER $P_{t h}$. When $s_{k}=0$ for $k<5$, according to (11), (28), and (41), $Y\left(s_{1}\right)$ is reduced to

$$
Y(\bar{\gamma} ; 0)=6\left(P_{64}(\bar{\gamma})-P_{t h}\right)
$$

where $P_{64}(\bar{\gamma})$ is the average BER of 64-QAM over a flat Rayleigh channel. The value of $Y(\bar{\gamma} ; 0)$ in (43) can be negative or positive, depending on the target BER $P_{t h}$.

We can observe in Fig. 5 that the solution of $Y\left(\bar{\gamma} ; \mathrm{s}\left(s_{1}\right)\right)=0$ is unique, when it exists. The locus of the minimum $Y\left(s_{1}\right)$, i.e., the trace curve of points $\left(Y_{\min }\left(s_{1, \min }\right), s_{1, \min }\right)$, where $Y$ has the minimum value, is also depicted in Fig. 5. The locus is always below the horizontal line of $Y\left(s_{1}\right)=0$ and asymptotically approaches this line, as the target BER $P_{t h}$ becomes smaller.

Fig. 6(a) depicts the switching levels optimized in this manner for our five-mode AQAM scheme maintaining the target average BERs of $P_{t h}=10^{-2}$.

The switching levels obtained using the per SNR-based Powell optimization method [18] are represented as the thin gray lines in Fig. 6(a) for comparison. In this case, all the modulation modes may be activated with a certain probability until the average SNR reaches the avalanche SNR value, while the scheme derived using Powell's optimization technique abandons the lower throughput modulation modes one by one as the average SNR increases. Fig. 6(b) depicts the average throughput $B$ of the AQAM scheme expressed in BPS, employing the switching levels optimized using the Lagrangian method. In Fig. 6(b), the average throughput of our six-mode 


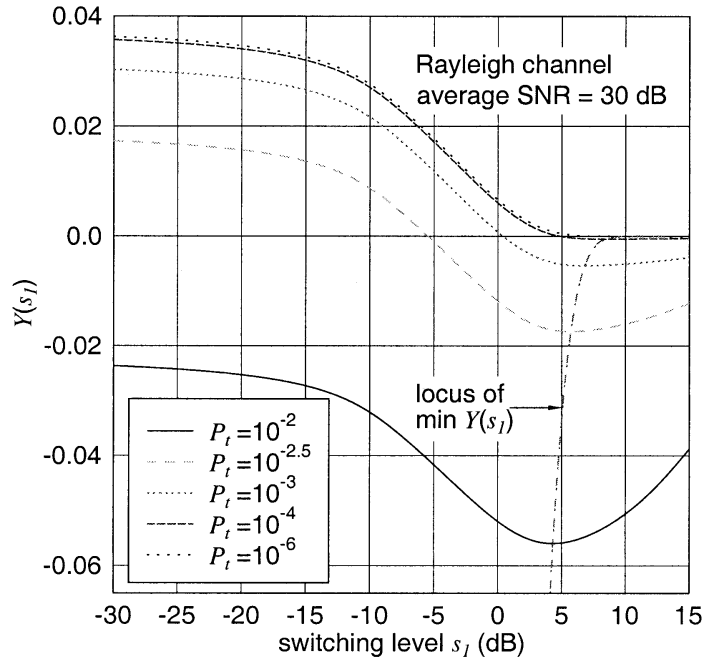

(a)

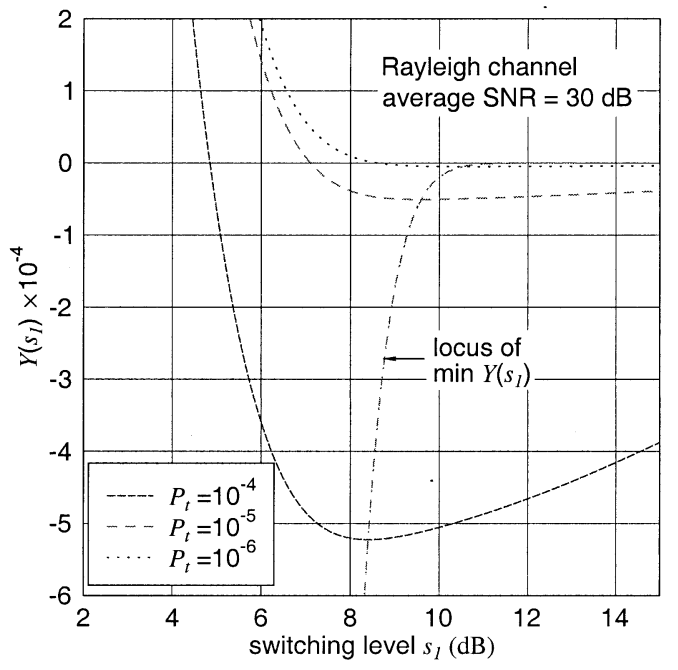

(b)

Fig. 5. The constraint function $Y\left(\bar{\gamma} ; \mathbf{s}\left(s_{1}\right)\right)$ defined in (41) for our five-mode AQAM scheme employing Gray-mapped square-constellation QAM operating over a flat Rayleigh-fading channel. The average SNR was $\bar{\gamma}=30 \mathrm{~dB}$ and it is seen that $Y$ has a single minimum value, while approaching $0^{-}$as $s_{1}$ increases. The solution of $Y\left(\bar{\gamma} ; \mathbf{s}\left(s_{1}\right)\right)=0$ exists when $Y(\bar{\gamma} ; 0)=6\left\{p_{64}(\bar{\gamma})-P_{t h}\right\}>0$ and is unique.

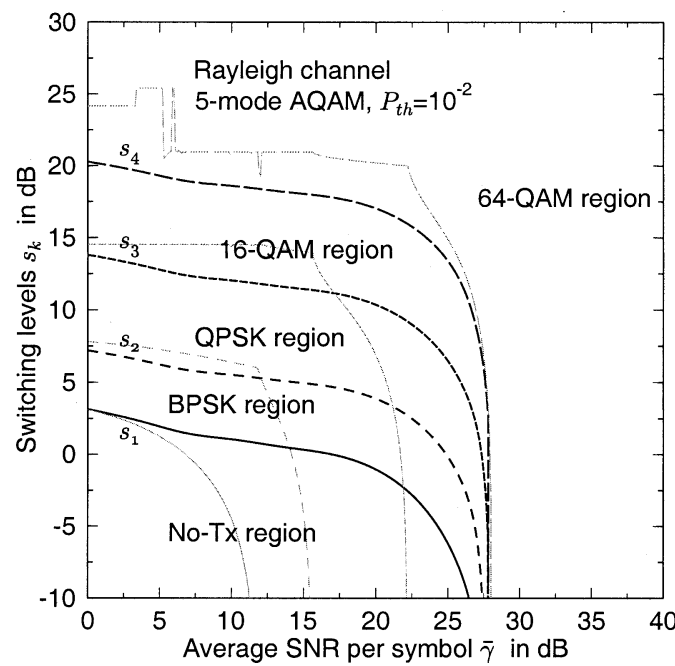

(a)

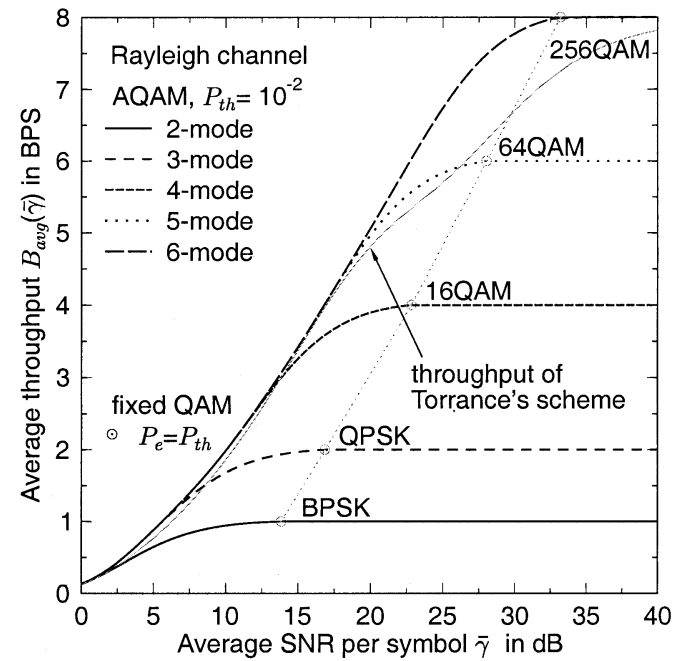

(b)

Fig. 6. The switching levels and the average BPS throughput of our five-mode AQAM scheme optimized at each average SNR value in order to achieve the target average BER of $P_{t h}=10^{-2}$ using the Lagrangian multiplier-based method. The switching levels based on per-SNR Powell's optimization [18] are represented in thin gray lines for comparison in (a). The average throughput of the six-mode AQAM scheme using Torrance's switching levels [16] is represented for comparison as the thin gray line in (b).

AQAM arrangement using Torrance's fixed-threshold based Powell-optimized scheme of [16] is represented as a thin gray line. The Lagrangian multiplier based scheme showed SNR gains of $0.6 \mathrm{~dB}, 0.5 \mathrm{~dB}, 0.2 \mathrm{~dB}$, and $3.9 \mathrm{~dB}$ for a BPS throughput of $1,2,4$, and 6 , respectively, compared to Torrance's scheme.

In conclusion, we derived an optimum mode-switching regime for a general adaptive-modulation scheme using the Lagrangian multiplier method and presented our numerical results for various AQAM arrangements. Since the results showed that the Lagrangian optimization-based scheme is superior in comparison to the other methods investigated, we will employ these switching levels in order to further investigate the performance of various adaptive-modulation schemes.

\section{RESULTS AND DISCUSSIONS}

\section{A. Narrow-Band Nakagami-m-Fading Channel}

In this section, we study the various aspects of the adaptive square QAM scheme, employing the optimum switching levels of Section III communicating over a narrow-band Nakagami- $m$ -fading channel. The pdf of the instantaneous channel SNR $\gamma$ of a system transmitting over the Nakagami-fading channel is given in (12) and the performance parameters characterizing the operation of the adaptive-modulation scheme were summarized in Section II-CI. The closed-form BER expressions of fixed-mode square QAM communicating over a Nakagami 


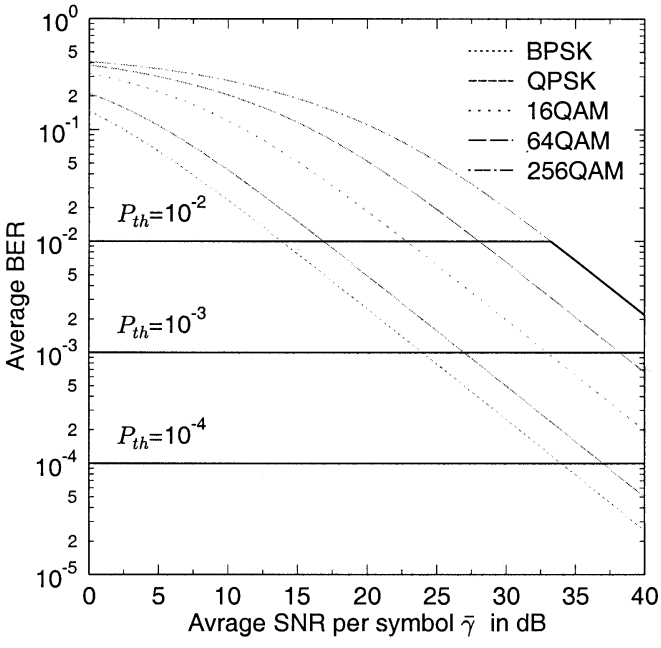

(a)

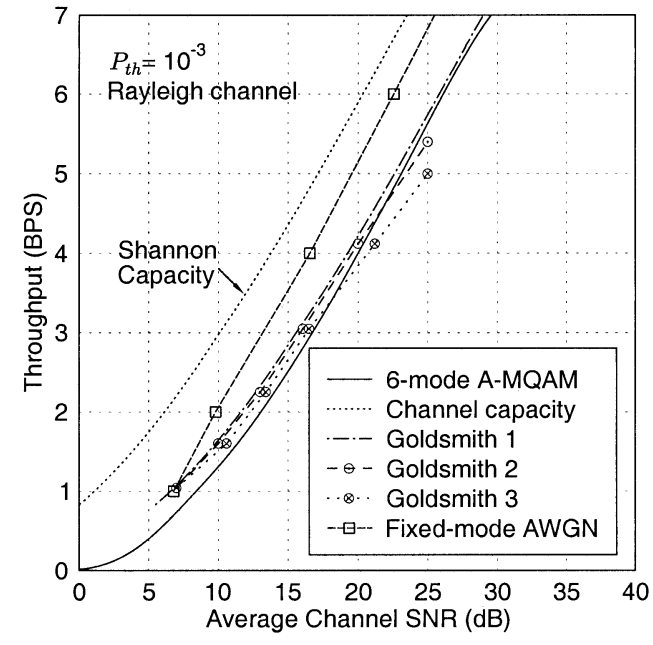

(b)

Fig. 7. The average BER and average throughput performance of a six-mode adaptive square QAM scheme operating over a flat Rayleigh channel $(m=1)$. (a) The constant target average BER is maintained over the entire range of the average SNR values up the avalanche SNR. (b) The average BPS throughput of the equivalent constant-power adaptive scheme is compared to to Goldsmith's schemes [15]. The "Goldsmith 1" and "Goldsmith 2" schemes represent a variable-power adaptive scheme employing a hypothetical continuously variable BPS QAM modulation modes and square QAM modes, respectively. The "Goldsmith 3" scheme represents the simulation results associated with the discrete-power adaptive square QAM scheme reported in [15].

channel can be expressed with the aid of [30, (14-4-15)] as

$$
\begin{aligned}
& P_{\text {MQAm }}(\bar{\gamma}) \\
& =\sum_{i} A_{i}\left[\frac{1}{2}\left(1-\mu_{i}\right)\right]_{j=0}^{m}\left(\begin{array}{c}
m-1+j \\
j
\end{array}\right)\left[\frac{1}{2}\left(1+\mu_{i}\right)\right]^{j}
\end{aligned}
$$

where $\mu_{i}$ is defined as

$$
\mu_{i} \triangleq \sqrt{\frac{a_{i} \bar{\gamma}}{2 m+a_{i} \bar{\gamma}}}
$$

and, again, the set of constants $\left\{A_{i}, a_{i}\right\}$ is given in Appendix A. The optimum switching levels of adaptive square QAM were studied in Section III as an example.

The average BER of our six-mode adaptive square QAM scheme operating over a flat Rayleigh-fading channel is depicted in Fig. 7(a), which shows that the modem maintains the required constant target BER until it reaches the BER curve of the specific fixed-mode modulation scheme employing the highest order modulation mode, namely 256 QAM. It then follows the BER curve of the 256-QAM mode. The various gray lines in the figure represent the BER of the fixed constituent modulation modes for transmission over a flat Rayleigh-fading channel. An arbitrarily low-target BER could be maintained at the expense of a reduced throughput.

The average throughput is shown in Fig. 7(b), together with the estimated channel capacity of the narrow-band Rayleigh channel [12] and [13], along with the throughput of several variable-power, variable-rate modems reported in [15]. Specifically, Goldsmith and Chua [15] studied the performance of their variable-power, variable-rate adaptive modems based on a BER bound of $m$-ary square QAM, rather than using an exact BER expression. Since our adaptive square QAM schemes do not vary the transmission power, our scheme can be regarded as a suboptimal policy [15]. However, the throughput performance of Fig. 7(b) shows that the SNR degradation is within $2 \mathrm{~dB}$ in the low SNR region and within half a decibel in the high-SNR region, in comparison to the ideal continuously variable-power adaptive QAM scheme employing a range of hypothetical continuously variable BPS throughput-based, rather than discrete-throughput-based QAM modes [15], represented as the "Goldsmith 1" scheme in the figure. Goldsmith and Chua [15] also characterized the performance of a variable-power, discrete-rate as well as that of a discrete-power, discrete-rate scheme, which we represented as the "Goldsmith 2" and "Goldsmith 3" schemes in Fig. 7(b), respectively. It can be seen in Fig. 7(b) that the optimum AQAM scheme outperforms the "Goldsmith 2" and "Goldsmith 3" schemes in the high-SNR region, even though we kept the power constant.

Fig. 8(a) depicts the average BPS throughput of our various adaptive square QAM schemes operating over a Rayleigh channel associated with $m=1$ at the target BER of $P_{t h}=10^{-3}$. Fig. 8(a) shows that even though the constituent modulation modes of our adaptive schemes do not include 3-, 5-, and 7-BPS constellations, the average BPS throughput steadily increases without undulations. Compared to the fixed-mode square QAM schemes operating over an AWGN channel, our adaptive schemes require additional SNRs of less than $3.5 \mathrm{~dB}$ when the throughput is below 6.5 BPS. Let us now investigate the effects of the Nakagami fading parameter $m$ on the average BPS throughput performance of the adaptive square QAM schemes, observing Fig. 8(b).The BPS throughput of the fixed-mode square QAM schemes for transmission over an AWGN channel is depicted in Fig. 8(b) as the ultimate performance limit achievable by the adaptive schemes operating over Nakagami-fading channels. As the Nakagami fading parameter $m$ increases from 1 to 2 and to 6 , the SNR gap between the adaptive schemes operating over a Nakagami-fading channel and the fixed-mode schemes operating over an AWGN channel decreases. When the average SNR is less than $\bar{\gamma} \leq 6 \mathrm{~dB}$, the average BPS throughput of our adaptive schemes decreases when the fading parameter $m$ increases. The rationale of this 


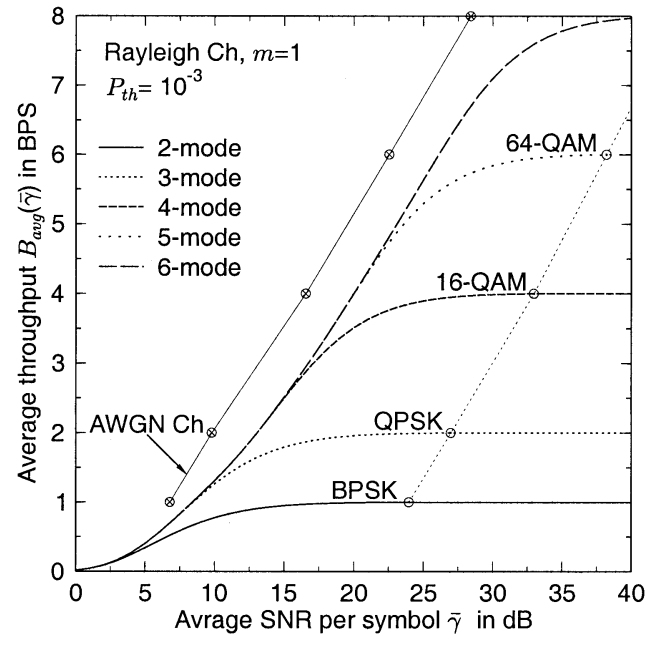

(a)

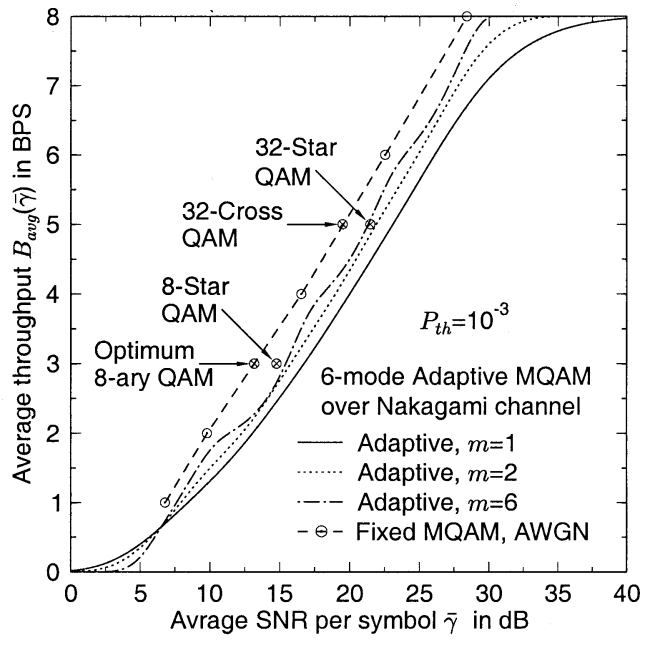

(b)

Fig. 8. The average BPS throughput of various adaptive-square QAM schemes operating over a Nakagami-fading channel at the target BER of $P_{t h}=10^{-3}$. (a) The fading severity parameter $m=1$. The markers " $\otimes$ " and " $\odot$ " represent the required SNR of the corresponding fixed-mode square QAM schemes achieving the same target BER as the adaptive schemes, operating over an AWGN channel and a Rayleigh channel, respectively. (b) As $m$ increases, the average throughput of the adaptive modem approaches the throughput of the corresponding fixed-square QAM modems operating over an AWGN channel.

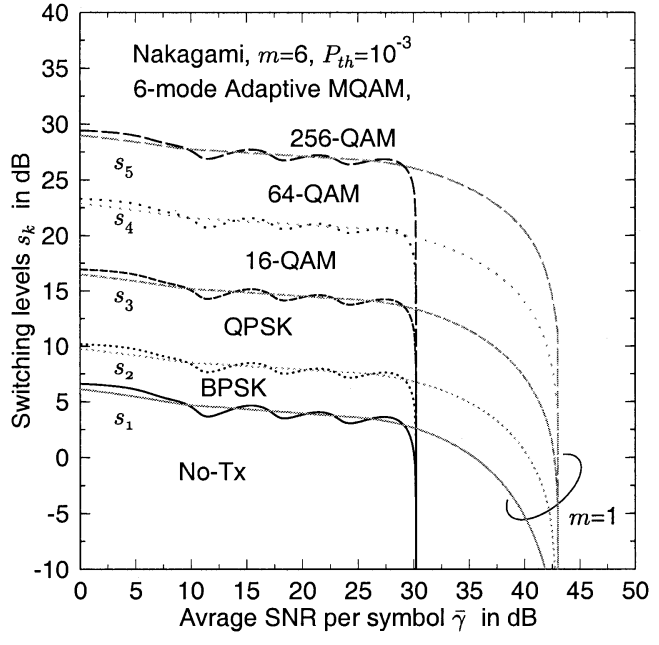

(a)

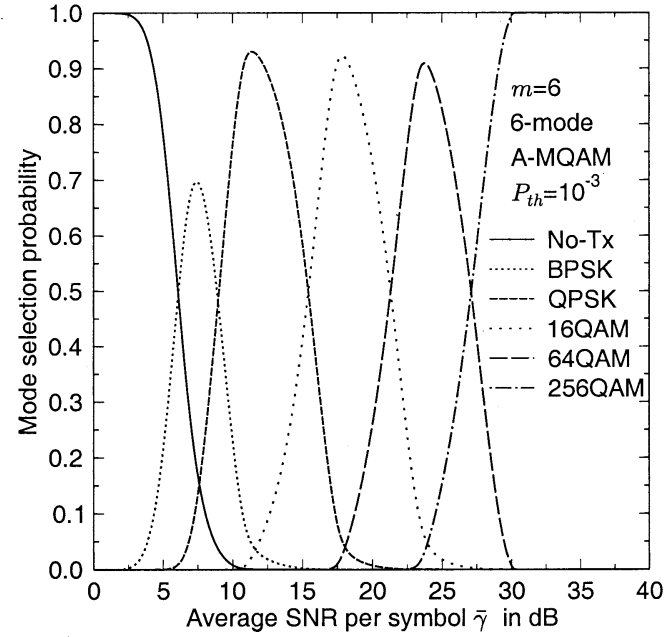

(b)

Fig. 9. The switching levels and the MPS of the six-mode adaptive-square QAM scheme operating over Nakagami-fading channels at the target BER $P_{t h}=$ $10^{-3}$. The bold lines are used for the fading parameter of $m=6$ and the gray lines are for $m=1$ in (a).

phenomenon is that as the channel becomes more and more like an AWGN channel due to increasing $m$ parameter, the probability of activating the BPSK mode is reduced, resulting in more frequent activation of the No-Tx mode and, hence, the corresponding average BPS throughput inevitably decreases. We can also observe that the average BPS throughput recorded for the fading parameter of $m=6$ exhibits an undulating curve. For example, an increased $m$ value results in a limited improvement of the corresponding average BPS throughput near the throughput values of 2.5, 4.5, and 6.5 BPS. This is because our adaptive square QAM schemes do not use 3-, 5-, and 7-BPS constituent modes. Fig. 9(a) depicts the corresponding optimum mode-switching levels for the six-mode adaptive square QAM scheme. The black lines represent the switching levels when the Nakagami fading parameter is $m=6$ and the gray lines when $m=1$. In general, the lower the switching levels, the higher the average BPS throughput of the adaptive modems. When the Nakagami fading parameter is $m=1$, the switching levels decrease monotonically, as the average SNR increases. However, when the fading severity parameter is $m=6$, the switching levels fluctuate, exhibiting several local minima around 12,18 , and $24 \mathrm{~dB}$. In general, the optimum switching levels become higher as the average SNR becomes higher. However, the switching level curves undulate because, as the average SNR becomes higher, the next higher throughput modulation mode becomes predominantly selected and the average target BER cannot be maintained unless the switching levels are increased. In the extreme case of $m \rightarrow \infty$, i.e., when operating over an AWGN-like channel, the switching levels would be $s_{1}=s_{2}=0$ and $s_{k}=\infty$ for other $k$ values in the SNR range of $9.8 \mathrm{~dB}<\bar{\gamma}<16.5 \mathrm{~dB}, s_{1}=s_{2}=s_{3}=0$ and $s_{4}=s_{5}=\infty$ when we have $16.5 \mathrm{~dB}<\bar{\gamma}<22.5 \mathrm{~dB}$, $s_{k}=0$ except for $s_{5}=\infty$ when the SNR is in the range of $22.5 \mathrm{~dB}<\bar{\gamma}<28.4 \mathrm{~dB}$. Finally, we have $s_{k}=0$ for 


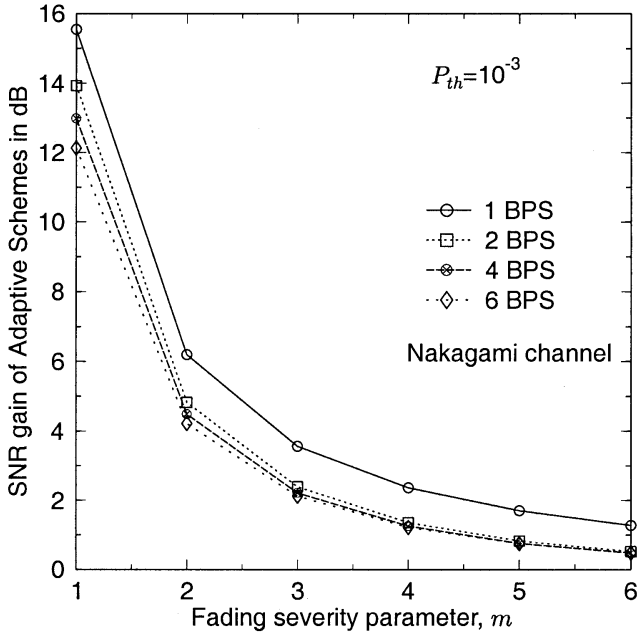

Fig. 10. The SNR gain of the six-mode adaptive-square QAM scheme in comparison to the various fixed-mode square QAM schemes yielding the same BPS throughput at the target BER of $P_{t h}=10^{-3}$. The performance advantage of the adaptive-square QAM schemes decreases as the fading becomes less severe.Fig. 13. Equivalent low-pass model of a $D$ th-order antenna diversity based RAKE receiver-assisted AQAM system.

$\forall k$, if $\bar{\gamma}>28.4 \mathrm{~dB}$, when considering the fixed-mode square QAM performance achieved for transmission over an AWGN channel represented by the makers " $\odot$ " in Fig. 8(b). Observing Fig. 9(b), we find that our adaptive schemes become highly "selective" when the Nakagami-fading parameter becomes $m=6$, exhibiting narrow triangular mode-selection pdf shapes. As $m$ increases, the shapes will eventually converge to Kronecker delta functions.

A possible approach to reducing the undulating behavior of the average BPS throughput curve is the introduction of a 3- and 5-BPS mode as additional constituent modem modes. The power-efficiency of 8-Star QAM and 32-Star QAM is insufficient for maintaining a linear growth of the average BPS throughput, as we can observe in Fig. 8(b). Instead, the most power-efficient twin-ring phasor-constellation based 8-ary QAM scheme [30, pp. 279] and 32-ary cross-shaped constellation based QAM scheme [2, p. 236] have the potential for reducing these undulation effects.

The effects of the Nakagami-fading factor $m$ on the relative SNR gain of our adaptive Square QAM scheme can be observed in Fig. 10. The less severe the fading, the smaller the relative SNR advantage of employing adaptive Square QAM in comparison to its fixed-mode counterparts. Except for the 1-BPS mode, the SNR gains become less than $0.5 \mathrm{~dB}$ when $m$ is increased to 6 at the target BER of $P_{t h}=10^{-3}$.

\section{B. Performance Over Narrow-Band Rayleigh Channels Using Antenna Diversity}

As we discussed in Section II-C1, the performance of the system when communicating over a flat Nakagami-fading channel having a fading parameter of $m$ is equivalent to that of the scheme transmitting over $D=m$ number of independent flat Rayleigh channels when maximum ratio combining (MRC)-assisted diversity is employed. Therefore, the results of Section IV-A can be invoked for characterizing an MRC antenna diversity-assisted adaptive-square QAM scheme operating over $D$ independent Rayleigh channels. However, the combined average SNR of the MRC assisted adaptive square QAM scheme is $D$ times higher than that of the adaptive square QAM scheme communicating over a flat Nakagami-fading channel. Hence, in order to gain an insight into the performance of the MRC antenna-diversity assisted adaptive square QAM scheme communicating over $D$ number of independent Rayleigh-fading channels, the related performance is presented in this section without repeating the results similar to those presented in Section IV-A.

The BER expression of the fixed-mode coherent BPSK scheme can be found in [30, p. 781] and those of coherent square QAM can be readily expressed using (44) and (45) with the substitution of $m \Rightarrow D$ and $\bar{\gamma} \Rightarrow D \bar{\gamma}$. The performance of antenna-diversity assisted adaptive square QAM schemes can be readily analyzed using the technique developed in Section III.

Fig. 11(a) depicts the average BPS throughput performance of our adaptive schemes employing MRC-aided antenna diversity [31, Ch. 5, 6] operating over independent Rayleigh-fading channels at the target average BER of $P_{t h}=10^{-3}$. The markers represent the performance of the corresponding fixed-mode square QAM modems in the same propagation scenario. The average SNRs that are required for achieving the target BER of the fixed-mode schemes and that of the adaptive schemes decrease as the antenna diversity order increases. However, the differences between the required SNRs of the adaptive schemes and their fixed-mode counterparts also decrease as the antenna diversity order increases. The SNRs of both schemes that are required for achieving the target BERs of $P_{t h}=10^{-3}$ are displayed in Fig. 11(b), where we can observe that employing dual antenna based diversity is sufficient for the fixed-mode schemes in order to obtain half of the achievable SNR gain of the six antenna-aided diversity scheme, whereas triple-antenna assisted diversity is required for the adaptive schemes operating in the same scenario.

\section{Performance Over Wide-Band Rayleigh Channels Using Antenna Diversity}

Wide-band fading channels are characterized by their multipath intensity profiles (MIPs). In order to study the performance of the various adaptive-modulation schemes, we employ two different MIP models in this section, namely a shortened wireless asynchronous transfer mode (W-ATM) channel [2, Ch. 20] characteristic of an indoor scenario and a bad-urban reduced-model A (BU-RA) channel [32], which is typical for a hilly urban outdoor scenario. Their MIPs are depicted in Fig. 12. The W-ATM channel exhibits short-range low-delay multipath components, while the BU-RA channel exhibits six higher delay multipath components. Again, let us assume that our receivers are equipped with MRC RAKE receivers [33], employing a sufficiently higher number of RAKE fingers in order to capture all the multipath components generated by our channel models. Furthermore, we employ antenna diversity [31, Ch. 5] at the receivers. This combined diversity scheme is often referred to as a two-dimensional (2-D) RAKE receiver [34, pp. 263]. 


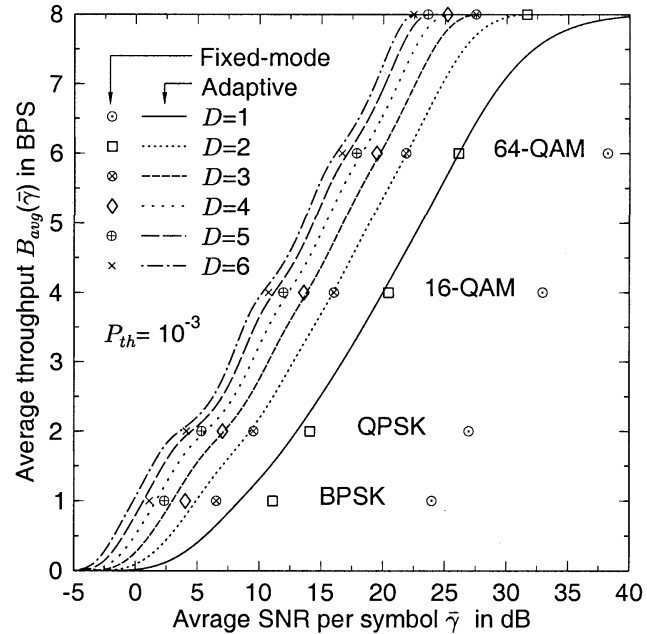

(a)

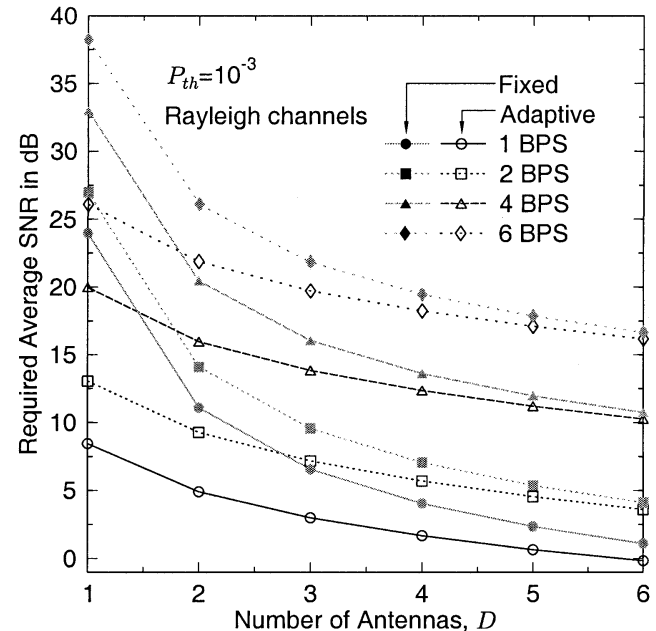

(b)

Fig. 11. The average BPS throughput and the required SNR of the MRC-aided antenna-diversity assisted adaptive-square QAM scheme operating over independent Rayleigh-fading channels at the target average BER of $P_{t h}=10^{-3}$. The markers represent the corresponding fixed-mode square QAM performances in (a).

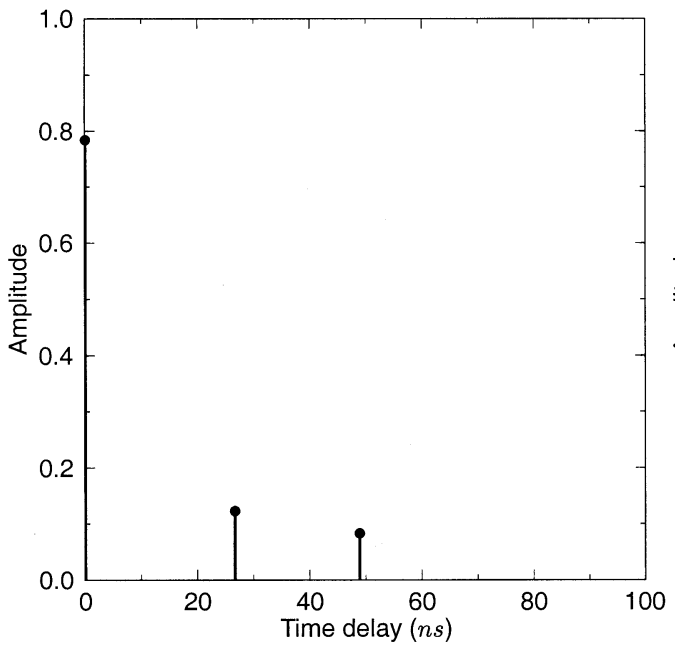

(a)

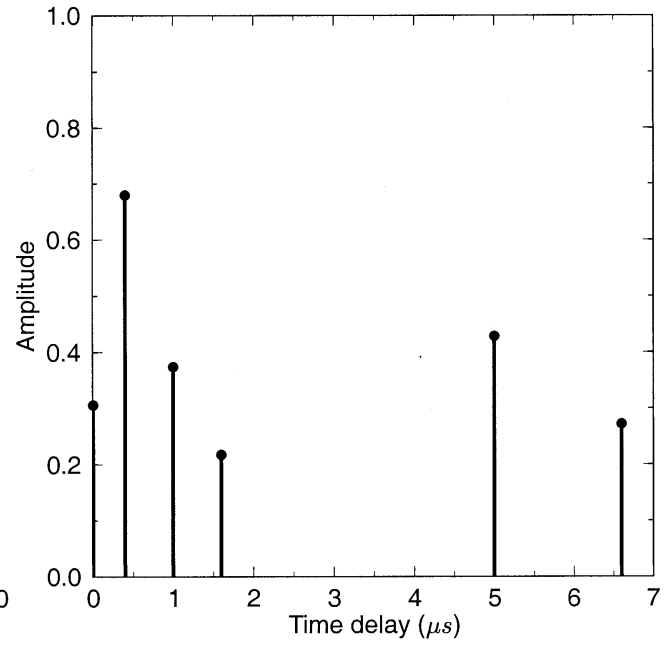

(b)

Fig. 12. MIPs of the wireless asynchronous transfer mode (W-ATM) indoor channel [2, Ch. 20] and that of the bad-urban reduced-model A (BU-RA) channel [32].

The schematic of our RAKE receiver and $D$ antenna diversity assisted adaptive quadrature amplitude modulation (AQAM) system is illustrated in Fig. 13. For the sake of analytical tractability we assume that each antenna experiences an identical MIP, while each diversity branch experiences independent Rayleigh fading. Our 2-D RAKE assisted fixed-mode QAM schemes are analyzed in Appendix E and the characteristic parameters of the 2-D RAKE assisted AQAM scheme are also given in Appendix F. Hence, the performance of our 2-D-RAKE assisted AQAM scheme employing the optimum switching levels can be readily characterized.

The average BPS throughputs of our 2-D-RAKE assisted adaptive schemes operating over the two different types of wide-band channel scenarios is presented in Fig. 14 for the target BER of $P_{t h}=10^{-3}$. The throughput performance depicted corresponds to the upper-bound performance of direct-sequence code division multiple access (DS-CDMA) or multicarrier CDMA employing RAKE receivers and that of the MRC-aided diversity-assisted scheme in the absence of multiple access interference (MAI). We can observe that the BPS throughput curves undulate when the number of antennas $D$ increases. This effect is more pronounced for transmission over the BU-RA channel, since this channel exhibits six multipath components, increasing the available diversity potential of the system approximately by a factor of two in comparison to that of the W-ATM channel. The performance of our adaptive scheme employing more than three antennas for transmission over the BU-RA channel could not be obtained owing to numerical instability, since the associated curves become similar to a series of step-functions, which is not analytic in mathematical terms.

The corresponding mode-switching levels and mode-selection probabilities are shown in Fig. 15. Again, the switching levels heavily undulate. The mode-selection probability curve of BPSK has a triangular shape, increasing linearly, as the average SNR $\bar{\gamma}$ increases to $2.5 \mathrm{~dB}$ and decreasing linearly 


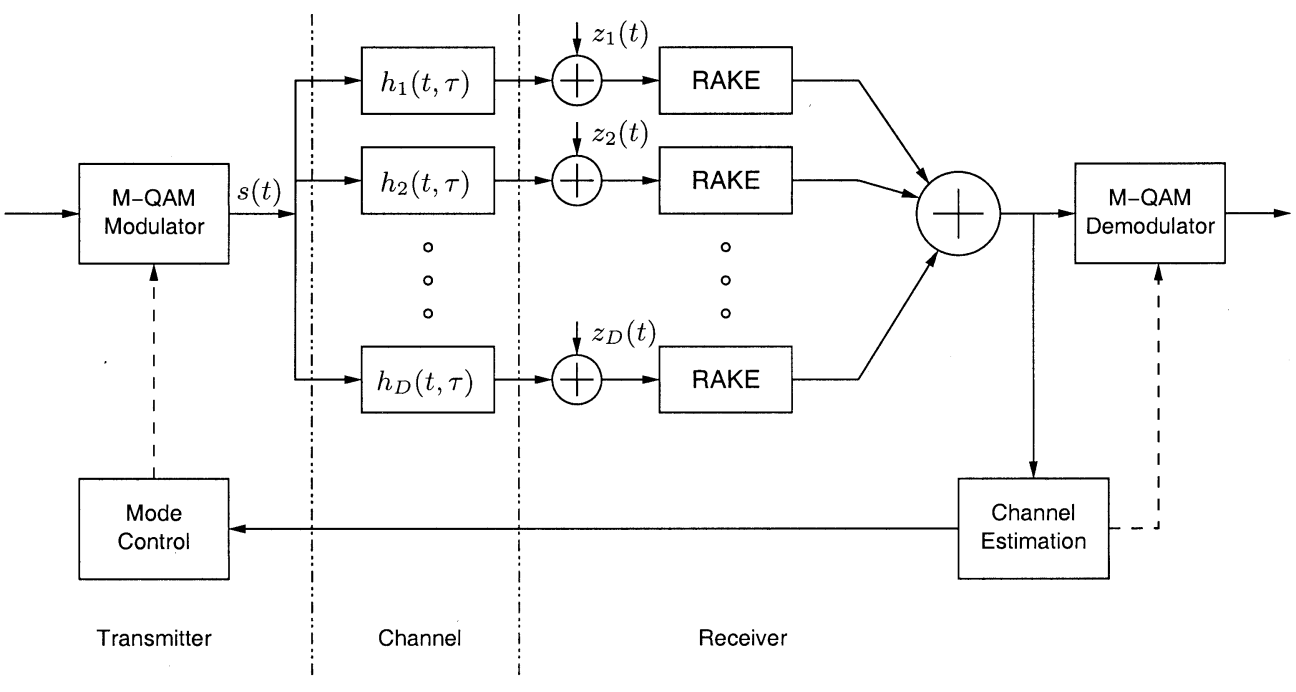

Fig. 13. Equivalent low-pass model of a $D$ th-order antenna diversity-based RAKE receiver-assisted AQAM system.

again, as $\bar{\gamma}$ increases beyond $2.5 \mathrm{~dB}$. On the other hand, the mode-selection probability curve of QPSK increases linearly and decreases exponentially, since no 3-BPS mode is used. This explains why the BPS throughput curves increase in a near-linear fashion in the SNR range of $0-5 \mathrm{~dB}$ and in a staircase fashion beyond that point. We may conclude that the staircase-like shape in the upper SNR range is a consequence of the absence of the 3-, 5-, and 7-BPS modulation modes in the set of constituent modulation modes employed. As we discussed in Section IV-A, this problem may be mitigated by introducing power-efficient twin-ring 8-QAM cross-constellation-based 32-QAM and 128-QAM modes.

The average SNRs required for achieving the target BER of $P_{t h}=10^{-3}$ by the 2-D-RAKE assisted adaptive scheme and the fixed-mode schemes operating over wide-band fading channels are depicted in Fig. 16. Since the fixed-mode schemes employing RAKE receivers are already enjoying the diversity benefit of multipath-fading channels, the SNR advantages of our adaptive schemes are less than 8 and $2.6 \mathrm{~dB}$ over the W-ATM and BU-RA channels, respectively, even when a single antenna is employed. This relatively small SNR gain, in comparison to those observed over narrow-band fading channels in Fig. 11(b), erode as the number of antennas increases. For example, when the number of antennas is $D=6$, the SNR gains of the adaptive schemes operating over the W-ATM channel of Fig. 12(a) become virtually zero, where the combined channel becomes an AWGN-like channel. On the other hand, $D=3$ number of antennas is sufficient for the BU-RA channel for exhibiting such a behavior since the underlying multipath diversity provided by the six-path BU-RA channel is higher than that of the tree-path W-ATM channel.

\section{Uncoded Adaptive Multicarrier Schemes}

The performance of the various adaptive square QAM schemes has also been studied in the context of multicarrier systems [2], [35], [36]. The family of orthogonal frequency-division multiplex (OFDM) [37] systems convert frequency-elective Rayleigh channels into frequency-nonselective or flat Rayleigh channels for each subcarrier, provided that the number of subcarriers is sufficiently high. The power and bit-allocation strategy of adaptive OFDM has attracted substantial research interests [2] and it was argued that OFDM is particularly suitable for combined time-frequency domain processing [36]. Since each subcarrier of an OFDM system experiences a flat Rayleigh channel, we can apply adaptive modulation for each subcarrier independently from other subcarriers. Moreover, a practical scheme would group the subcarriers into similar-quality subbands for the sake of reducing the associated modem mode-signaling requirements. The performance of this AQAM-assisted OFDM (A-OFDM) scheme is identical to that of the adaptive scheme operating over flat Rayleigh-fading channels, characterized in Section IV-B.

The MC-CDMA [38], [39] receiver can be regarded as a frequency domain RAKE-receiver, where the multiple carriers play a role similar to that of the time-domain RAKE fingers. Our simulation results showed that the single-user BER performance of MC-CDMA employing multiple antennae is essentially identical to that of the time-domain RAKE receiver using antenna diversity, provided that the spreading factor is higher than the number of resolvable multipath components in the channel. Hence, the throughput of the RAKE-receiver over the three-path W-ATM channel [2] and the six-path BU-RA channel [32] studied in Section IV-C can be used for investigating the upper-bound performance of adaptive MC-CDMA schemes when communicating over these channels. Fig. 17 compares the average BPS throughput performances of these schemes, where the throughput curves of the various adaptive schemes are represented as three different types of lines, depending on the underlying channel scenarios, while the fixed-mode schemes are represented as three different types of markers. The solid line corresponds to the performance of A-OFDM and the marker " $\bullet$ " corresponds to that of the fixed-mode OFDM. On the other hand, the dotted lines correspond to the BPS throughput performance of adaptive MC-CDMA operating over wide-band channels and the 


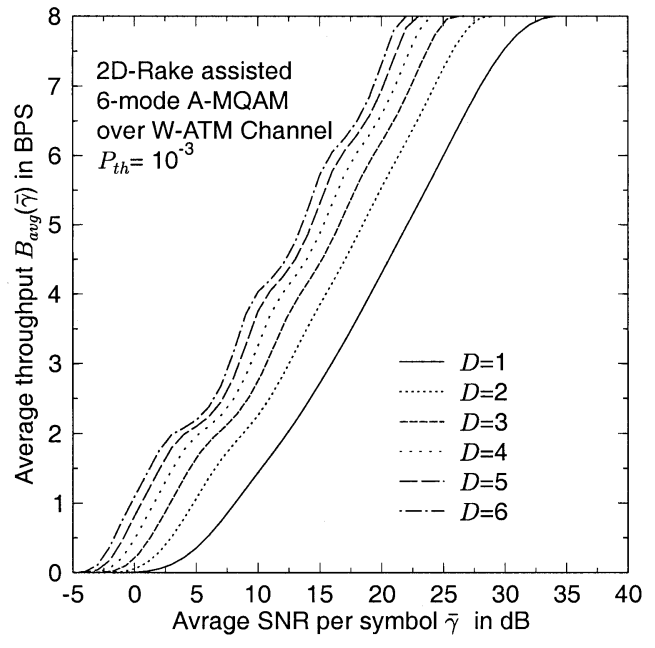

(a)

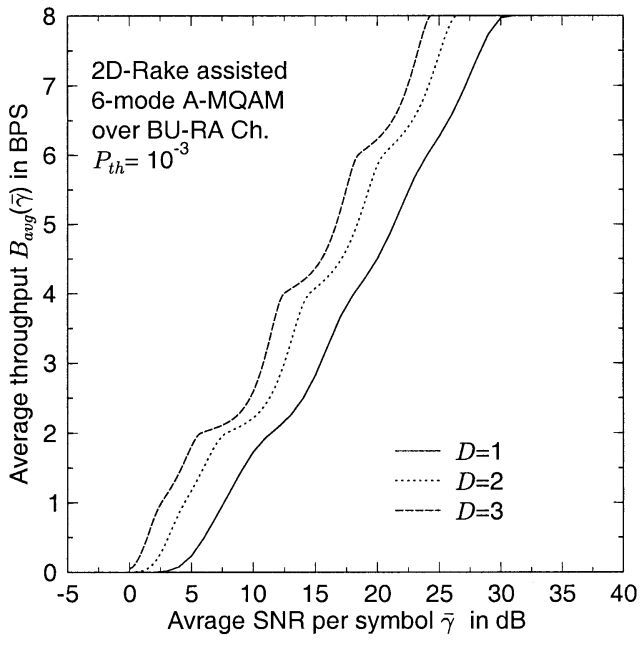

(b)

Fig. 14. The effects of the number of diversity antennas on the average BPS throughput of the 2-D RAKE assisted six-mode adaptive square QAM scheme operating over the wideband independently. Rayleigh-fading channels characterized in Fig. 9 at the target BER of $P_{t h}=10^{-3}$. The number of diversity antennas is $D$.
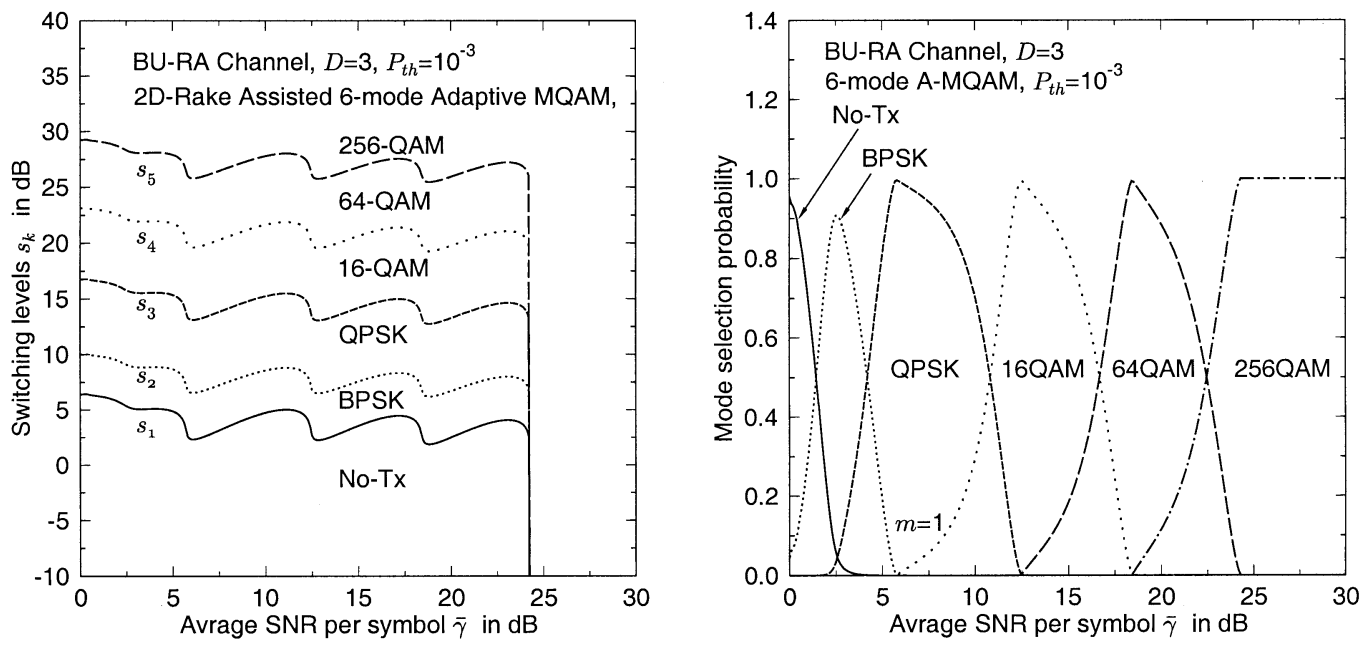

Fig. 15. The mode-switching levels and mode-selection probability of the 2-D RAKE assisted six-mode adaptive square QAM scheme using $D=3$ antennas operating over the BU-RA channel characterized in Fig. 13 at the target BER of $P_{t h}=10^{-3}$.

markers " $\odot$ " and " $\otimes$ " to those of the fixed-mode MC-CDMA schemes.

It can be observed that fixed-mode MC-CDMA has the potential to outperform A-OFDM, when the underlying channel provides sufficient diversity due to the high number of resolvable multipath components. For example, the performance of fixed-mode MC-CDMA operating over the W-ATM channel of Fig. 12(a) is slightly lower than that of A-OFDM for the BPS range of less than or equal to 6 BPS, owing to the insufficient diversity potential of the wide-band channel. On the other hand, fixed-mode MC-CDMA outperforms A-OFDM when the channel is characterized by the BU-RA model of Fig. 12(b). We have to consider several factors in order to resolve whether fixed-mode MC-CDMA is capable of outperforming A-OFDM. Firstly, fully loaded MC-CDMA, which can transmit the same number of symbols as OFDM, suffers from multicode interference and our simulation results showed that the SNR degrada- tion is about $2-4 \mathrm{~dB}$ at the BER of $10^{-3}$, when the minimum mean square error block decision feedback equalizer (MMSEBDFE) [40] based joint detector is used at the receiver. Considering these SNR degradations, the throughput of fixed-mode MC-CDMA using the MMSE-BDFE joint detection receiver falls just below that of the A-OFDM scheme when the channel is characterized by the BU-RA model. On the other hand, the adaptive schemes may suffer from inaccurate channel estimation/prediction and modem mode signaling feedback delay [15]. Hence, the preferred order of the various schemes may depend on the channel scenario encountered, on the interference effects, and on other practical issues, such as the aforementioned channel estimation accuracy, feedback delays, etc. In the previous sections, we have studied various receiver diversity-assisted adaptive single- and multicarrier modems. In the next section, we focus our attention on employing transmit-diversity assisted adaptive transceivers and channel coding. 


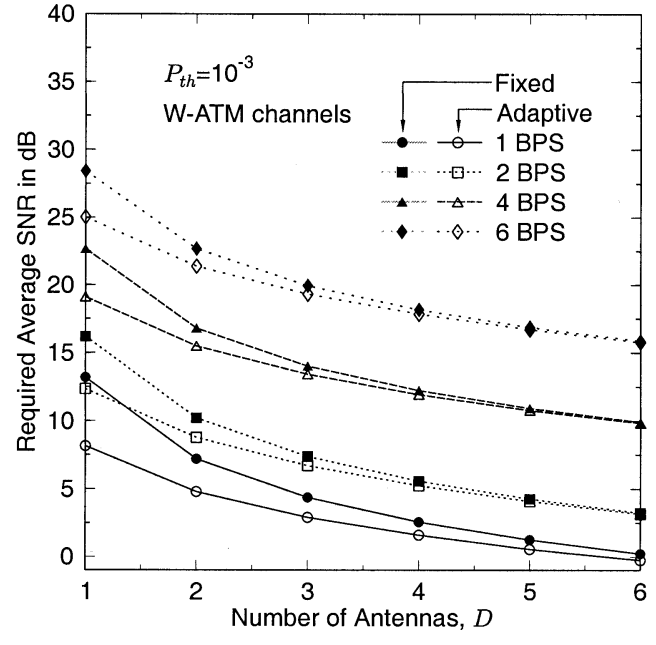

(a)

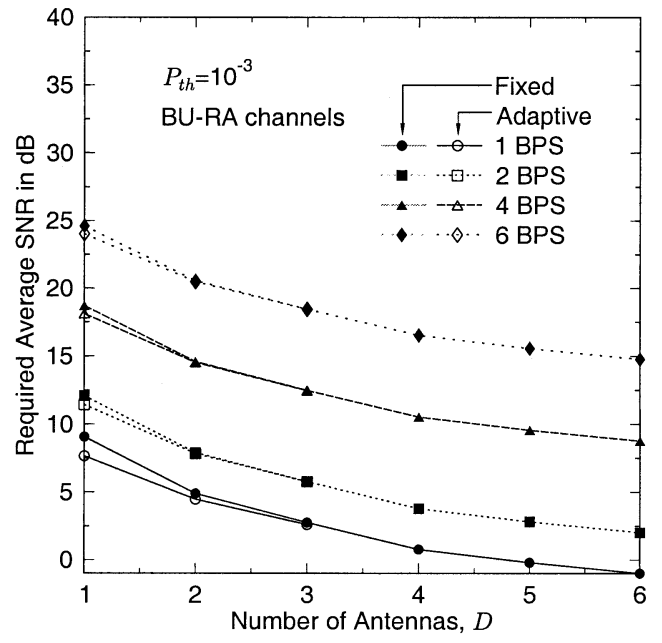

(b)

Fig. 16. The average SNRs required for achieving the target BER of $P_{t h}=10^{-3}$ by the 2-D RAKE assisted adaptive schemes and by the fixed-mode schemes operating over (a) the W-ATM channel and (b) the BU-RA channel.

\section{E. Concatenated Space-Time Block-Coded and Turbo-Coded Symbol-by-Symbol Adaptive OFDM and Multicarrier CDMA}

In the previous sections, we studied the performance of uncoded adaptive schemes. Since a forward error correction (FEC) code reduces the SNR required for achieving a given target BER at the expense of a reduced BPS throughput, it is interesting to investigate the performance of adaptive schemes employing FEC techniques [41]. A variety of FEC techniques have been used in the context of adaptive-modulation schemes [4], [41]. In their pioneering work on adaptive modulation, Webb and Steele [6] used a set of binary Bose-Chaudhuri-Hocquenghen (BCH) codes. Vucetic [42] employed various punctured convolutional codes in response to the time-variant channel's status. On the other hand, various trellis coded modulation (TCM) [41], [43], [44] schemes were used in the context of adaptive modulation by Alamouti and Kallel [45], Goldsmith and Chua [46], as well as by Hole et al. [47]. The comparative study of TCM, turbo TCM, bit-interleaved coded modulation (BICM), and iteratively detected BICM was provided in [41], [48]. Keller et al. studied the performance of redundant residue number system (RRNS) codes in the context of adaptive multicarrier modulation [2], [49], [50]. Various turbo-coded adaptive-modulation schemes have been investigated also by Liew et al. [4], [41], [51]-[53]. With the advent of space-time (ST) coding techniques [41], [54]-[56], various concatenated coding schemes combining ST and FEC coding can be applied in adaptive-modulation schemes [41]. In this section, we investigate the performance of various concatenated ST, block-coded, and turbo-coded adaptive OFDM [2], [4], [41] and MC-CDMA schemes.

Fig. 18 portrays the stylized transmitter structure of our system. The source bits are channel coded by a half-rate turbo convolutional encoder [41], [57] using a constraint length of $K=3$ as well as turbo interleaver having a memory of $L=3072$ bits and channel interleaved by a random block interleaver. Then, the AQAM block selects a modulation mode from the set of no transmission, BPSK, QPSK, 16-QAM, and 64-QAM, depending on the instantaneous channel quality perceived by the receiver, according to the SNR-dependent

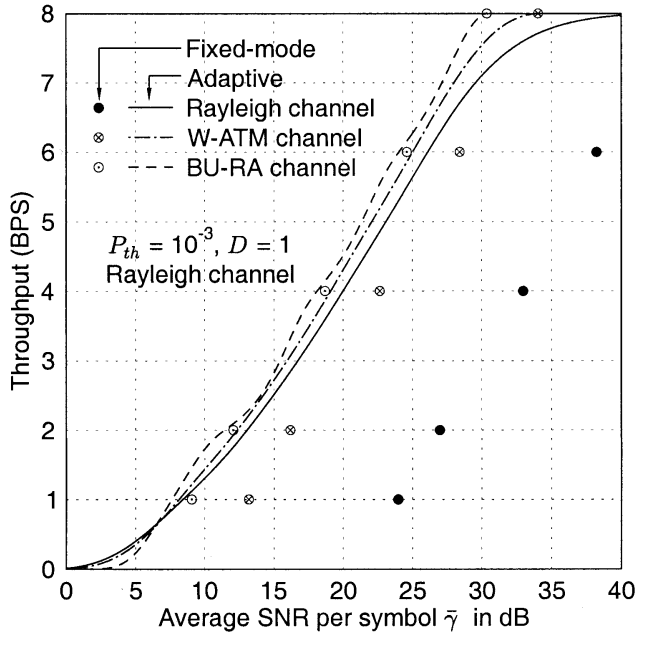

Fig. 17. The average BPS throughput of the adaptive schemes and fixed-mode schemes transmission over a narrow-band Rayleigh channel, the W-ATM channel, and BU-RA channel of Fig. 9 at the target BER of $P_{t h}=10^{-3}$.

optimum switching levels derived in Section III. It is assumed that the perfectly estimated channel quality experienced by receiver A is fed back to transmitter B superimposed on the next burst transmitted to receiver B of Fig. 1. The modulation mode-switching levels of our AQAM scheme determine the average BER as well as the average throughput.

The modulated symbol is now ST encoded. As seen at the bottom of Fig. 18, Alamouti's ST block code [41] and [55] is applied across the frequency domain, since it was found in [41] and [58] that from a wide-ranging set of combined channel coding and ST coding schemes, this particular combination exhibited the largest coding gain at a given complexity and at an effective throughput of 2- and 3-BPS. A pair of the adjacent subcarriers belonging to the same ST encoding block is assumed to have the same channel quality. We employed a wireless asynchronous transfer mode (W-ATM) channel model [2, p. 474] transmitting at a carrier frequency of $60 \mathrm{GHz}$, at a sampling rate of $225 \mathrm{MHz}$, and employing 512 subcarriers. Specifically, 


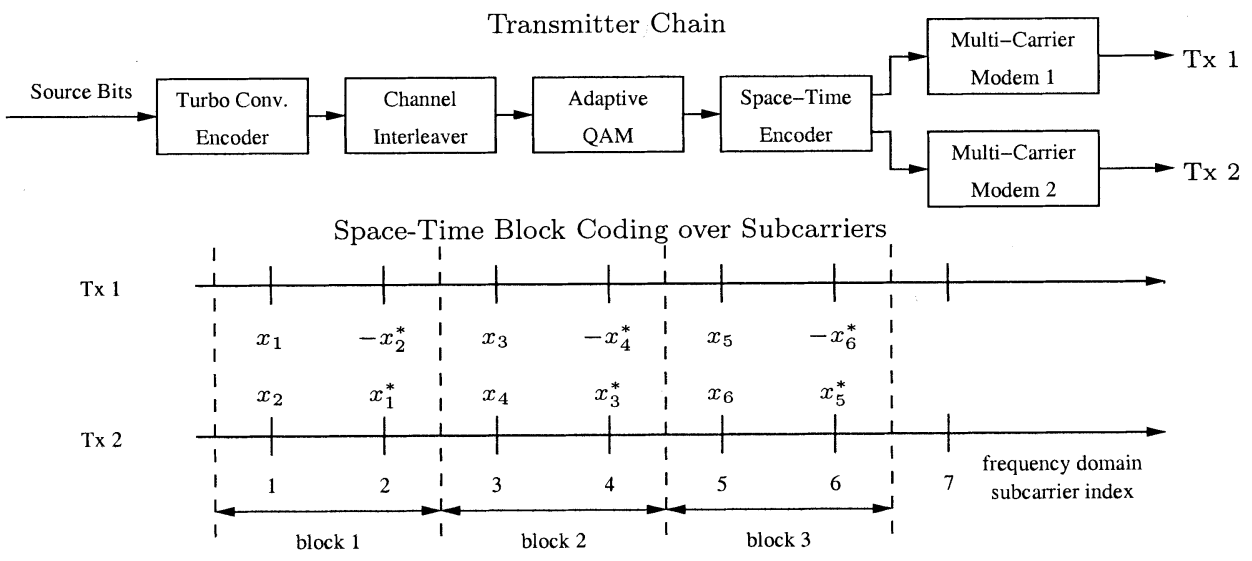

Fig. 18. Transmitter structure and space-time block encoding scheme.

we used a three-path fading channel model, where the average SNR of each path is given by $\bar{\gamma}_{1}=0.79192 \bar{\gamma}, \bar{\gamma}_{2}=0.12424 \bar{\gamma}$ and $\bar{\gamma}_{3}=0.08384 \bar{\gamma}$. The MIP of the W-ATM channel is illustrated in Fig. 12(a) in Section IV-C. Each of the transmit-diversity channels associated with a different antenna is assumed to exhibit independent Rayleigh fading.

The simulation results related to our uncoded adaptive modems are presented in Fig. 19. In Fig. 19(a), both the continuous black lines and the related markers drawn in continuous lines represent the BERs, while their gray or "halftone" counterparts the BPS throughputs. Viewing Fig. 19(a) from a different perspective, all the lines indicate analytical results, while all the markers correspond to simulation results. The set of three BER curves represented by the black lines that are decreasing as the average SNR increases corresponds to those of fixed-mode OFDM and the MC-CDMA schemes supporting $U=1$ and 16 users. Since we employed the optimum-switching levels derived in Section III, both our adaptive OFDM (AOFDM) and adaptive single-user MC-CDMA (AMC-CDMA) modems maintain the constant target BER of $10^{-3}$ up to the "avalanche" SNR value and then follow one of the three BER curves corresponding to the fixed 64-QAM mode. However, "full-user" AMC-CDMA, which is defined as an AMC-CDMA system supporting $U=16$ users with the aid of a spreading factor of $G=16$ and employing the MMSE-BDFE joint detection (JD) receiver [4] and [40], exhibits a slightly higher average BER than the BER target of $P_{t h}=10^{-3}$ due to the fact that the residual multiuser interference (MUI) of the imperfect joint detector does not strictly follow a Gaussian distribution. Since in Section III we derived the optimum-switching levels based on a single-user system free from MUI, the levels are no longer optimum when the residual MUI does not follow the Gaussian distribution. The average throughputs of the various schemes expressed in terms of BPS steadily increase and at high SNRs reach the throughput of 64 QAM, namely 6BPS. The throughput degradation of "full-user" MC-CDMA, imposed by the imperfect JD, was within a fraction of a decibel. Observe in Fig. 19(a) that the analytical and simulation results are in good agreement, which we denoted by the lines and distinct symbols, respectively.

The effects of ST coding on the average BPS throughput are displayed in Fig. 19(b). Specifically, the four different styles of thick lines represent the average BPS throughput of our AMC-CDMA scheme, while the four thin lines represent those of our AOFDM modem. The four pairs of hollow and filled markers associated with the four different ST-coded AOFDM and AMC-CDMA scenarios considered represent the BPS throughput versus SNR values associated with the fixed-mode OFDM and fixed-mode MMSE-BDFE JD-assisted MC-CDMA schemes. Specifically, observe for each of the 1,2, and 4 BPS fixed-mode schemes that the right-most markers, namely the circles, correspond to the $1-\mathrm{Tx} / 1-\mathrm{Rx}$ scenario, the squares to the $2-\mathrm{Tx} / 1-\mathrm{Rx}$ scheme, the triangles to the $1-\mathrm{Tx} / 2-\mathrm{Rx}$ arrangement, and the diamonds to the $2-\mathrm{Tx} / 2-\mathrm{Rx}$ scenarios. First of all, we can observe that the BPS throughput curves of OFDM and single-user MC-CDMA are close to each other, namely within $1 \mathrm{~dB}$ for most of the SNR range. This is surprising, considering that the fixed-mode MMSE-BDFE JD-assisted MC-CDMA scheme was reported to exhibit around $10 \mathrm{~dB}$ SNR gain at a BER of $10^{-3}$ and $30 \mathrm{~dB}$ gain at a BER of $10^{-6}$ over OFDM [59]. This is confirmed in Fig. 19(b) by observing that the SNR difference between the $\circ$ and $\bullet$ markers is approximately $10 \mathrm{~dB}$, regardless whether the 4 , 2 , or 1 BPS scenario is concerned.

Let us now compare the achievable SNR gains of the adaptive modems over the fixed modems. The SNR difference between the BPS curve of AOFDM and the fixed-mode OFDM represented by the symbol $\circ$ at the same throughput is approximately $15 \mathrm{~dB}$. The corresponding SNR difference between the adaptive and fixed-mode 4, 2, or 1 BPS MC-CDMA modem is approximately $5 \mathrm{~dB}$. More explicitly, since in the context of the W-ATM channel model [2, p. 474] fixed-mode MC-CDMA appears to exhibit a 10-dB SNR gain over fixed-mode OFDM, the additional 5-dB SNR gain of AMC-CDMA over its fixed-mode counterpart results in a total SNR gain of $15 \mathrm{~dB}$ over fixed-mode OFDM. Hence, ultimately, the performance of AOFDM and AMC-CDMA becomes similar.

Let us now examine the effect of ST block coding. The SNR gain of the fixed-mode schemes, due to the introduction of a 2-Tx/1-Rx ST block code, is represented as the SNR difference between the two right-most markers, namely circles and squares. These gains are nearly $10 \mathrm{~dB}$ for fixed-mode OFDM, while they are only $3 \mathrm{~dB}$ for the fixed-mode MC-CDMA modems. However, the corresponding gains are less than 


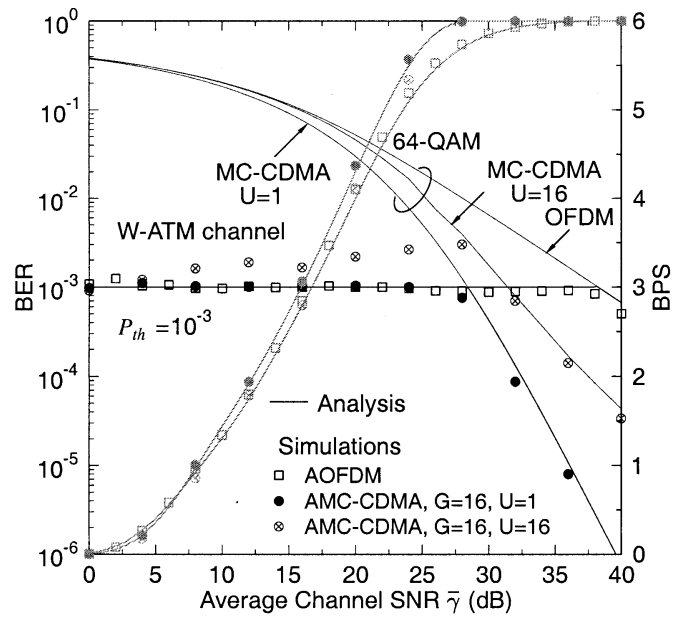

(a)

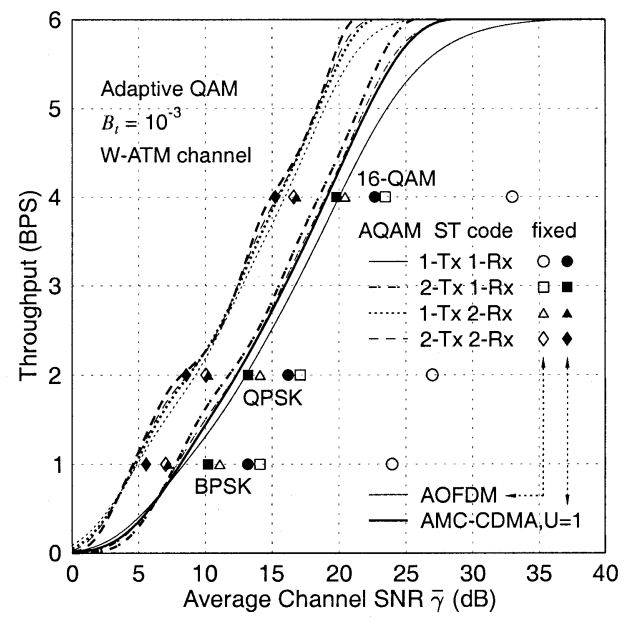

(b)

Fig. 19. Performance of uncoded five-mode AOFDM and AMC-CDMA. The target BER is $P_{t h}=10^{-3}$ when transmitting over the W-ATM channel [2, pp. 474]. (a) The constant average BER is maintained for AOFDM and single-user AMC-CDMA, while "full-user" AMC-CDMA exhibits a slightly higher average BER due to the residual MUI. (b) The SNR gain of the adaptive modems decreases as ST coding increases the diversity order. The BPS curves appear in pairs, corresponding to AOFDM and AMC-CDMA - indicated by the thin and thick lines, respectively - for each of the four different ST code configurations. The markers represent the SNRs required by the fixed-mode OFDM and MC-CDMA schemes for maintaining the target BER of $10^{-3}$ in conjunction with the four ST-coded schemes considered.

$1 \mathrm{~dB}$ for both adaptive modems, namely for AOFDM and AMC-CDMA. Since the transmitter power is halved due to using two Tx antennas in the ST code, a 3-dB channel SNR penalty was already applied to the curves in Fig. 19(b). The introduction of a second receive antenna instead of a second transmit antenna eliminates this $3-\mathrm{dB}$ penalty, which results in a better performance for the 1-Tx/2-Rx scheme than for the $2-T x / 1-R x$ arrangement. Finally, the $2-T x / 2-R x$ system gives approximately 3-4 dB SNR gain in the context of fixed-mode OFDM and a 2-3 dB SNR gain for fixed-mode MC-CDMA, in both cases over the 1-Tx/2-Rx system. By contrast, the SNR gain of the 2-Tx/2-Rx scheme over the 1-Tx/2-Rx based adaptive modems was, again, less than $1 \mathrm{~dB}$ in Fig. 19(b). More importantly is, for the $2-\mathrm{Tx} / 2-\mathrm{Rx}$ scenario, the advantage of employing adaptive-modulation erodes, since the fixed-mode MC-CDMA modem performs as well as the AMC-CDMA modem in this scenario. Moreover, the fixed-mode MC-CDMA modem still outperforms the fixed-mode OFDM modem by about $2 \mathrm{~dB}$. We conclude that since the diversity-order increases with the introduction of ST block codes, the channel quality variation becomes sufficiently small for the performance advantage of adaptive modems to erode. This is achieved at the expense of a higher complexity, due to employing two transmitters and two receivers in the ST-coded system.

The performance of the concatenated ST block coded and turbo convolutional coded adaptive modems is depicted in Fig. 20. We applied the optimum set of switching levels designed in Section III for achieving an uncoded BER of $3 \times 10^{-2}$. This uncoded target BER was stipulated after observing that it is reduced by the half-rate, $K=3$ turbo convolutional coding to a BER below $10^{-7}$, when transmitting over AWGN channels. However, our simulation results yielded zero bit errors when transmitting $10^{9}$ bits, except for some SNRs when employing only a single antenna.

Fig. 20(a) shows the BER of our turbo-coded adaptive modems, when a single transmit and receive antenna is used.
We observe in the figure that the BER reaches its highest value around the "avalanche" SNR point, where the adaptive-modulation scheme consistently activates 64 QAM. The system is most vulnerable around this point. In order to interpret this phenomenon, let us briefly consider the associated channel interleaving aspects. For practical reasons, we have used a fixed turbo interleaver length of $L=3072$ bits. When the instantaneous channel quality was high, the $L=3072$ bits were spanning a shorter time-duration during their passage over the fading channel, corresponding to a reduced number of modulated symbols since the effective BPS throughput was high. Hence, the channel errors appeared more bursty than in the lower-throughput AQAM modes, which conveyed the $L=3072$ bits over a longer time duration, corresponding to a higher number of modulated symbols and, hence, dispersing the error bursts over a longer duration of time. The uniform dispersion of erroneous bits versus time enhances the error-correction power of the turbo code. On the other hand, in the SNR region beyond the "avalanche" SNR point seen in Fig. 20(a), the system exhibited a lower uncoded BER, reducing the coded BER even further. This observation suggests that further research ought to determine the set of switching thresholds directly for a coded adaptive system, rather than by simply estimating the uncoded BER, which is expected to result in nearly error-free transmission.

We can also observe in Fig. 20(a) that the turbo-coded BER of AOFDM is higher than that of AMC-CDMA in the SNR rage of $10-20 \mathrm{~dB}$, even though the uncoded BER is the same. This appears to be the effect of the limited exploitation of frequency domain diversity of coded OFDM, compared to MC-CDMA, which leads to a more bursty uncoded error distribution, hence degrading the turbo-coded performance. The fact that ST block coding aided multiple antenna systems shows virtually error-free performance corroborates our argument.

Fig. 20(b) compares the throughputs of the coded adaptive modems and the uncoded adaptive modems exhibiting a com- 


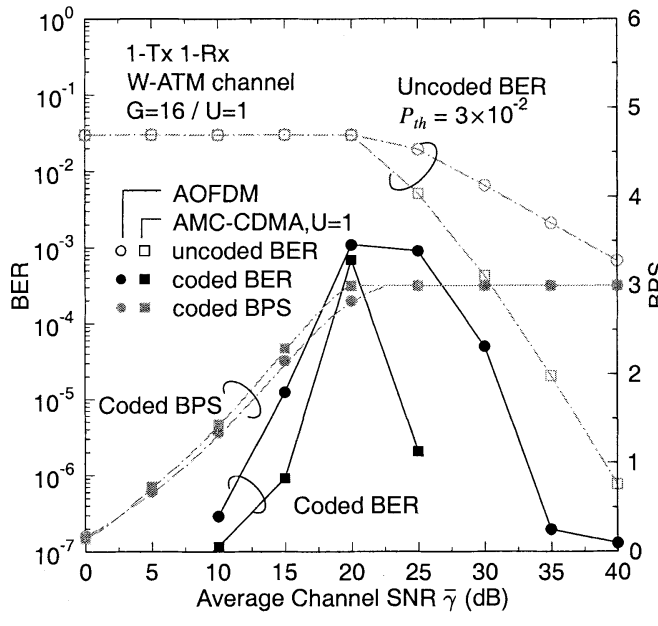

(a)

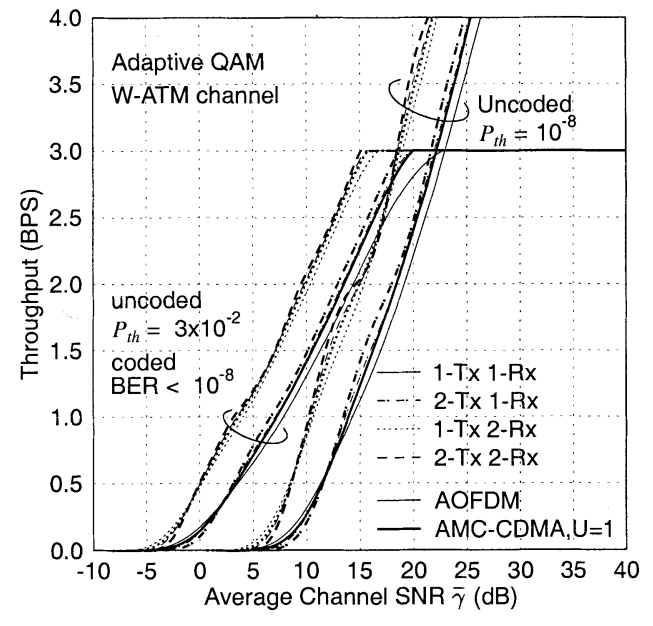

(b)

Fig. 20. Performance of the concatenated ST block-coded and turbo-convolutional-coded adaptive OFDM and MC-CDMA systems over W-ATM channel of [2, p. 474]. The uncoded target BER is $P_{t h}=3 \times 10^{-2}$. The coded BER was less than $10^{-8}$ for most of the SNR range, resulting in virtually error-free transmission. (a) The coded BER becomes higher near the "avalanche" SNR point when a single antenna was used. (b) The coded adaptive modems have SNR gains up to $7 \mathrm{~dB}$ compared to their uncoded counterparts, achieving a comparable average BER.

parable average BER. The SNR gains due to channel coding were in the range of $0-8 \mathrm{~dB}$, depending on the SNR region and on the scenarios employed. Each bundle of throughput curves corresponds to the scenarios of 1-Tx/1-Rx OFDM, 1-Tx/1-Rx MC-CDMA, 2-Tx/1-Rx OFDM, 2-Tx/1-Rx MC-CDMA, 1-Tx/2-Rx OFDM, 1-Tx/2-Rx MC-CDMA, 2-Tx/2-Rx OFDM, and 2-Tx/2-Rx MC-CDMA, starting from the far right curve, when considered for throughput values higher than 0.5 BPS. The SNR difference between the throughput curves of the ST and turbo coded AOFDM and those of the corresponding AMC-CDMA schemes was reduced compared to the uncoded performance curves of Fig. 19(b). The SNR gain owing to ST block coding assisted transmit diversity in the context of AOFDM and AMC-CDMA was within $1 \mathrm{~dB}$ due to the halved transmitter power. Therefore, again, ST block coding appears to be less effective in conjunction with adaptive modems. Phrased from a different perspective, the advantages of adaptive-modulation erode in conjunction with multiple transmitters and receivers since both techniques attempt to mitigate the effects of channel quality fluctuations.

In conclusion, the performance of ST block coded constant-power adaptive multicarrier modems employing optimum SNR-dependent modem mode-switching levels were investigated in this section. The adaptive modems maintained the constant target BER stipulated, while maximizing the average throughput. As expected, it was found that ST block coding reduces the relative performance advantage of adaptive modulation, since it increases the diversity order and eventually reduces the channel quality variations. When turbo convolutional coding was concatenated to the ST block codes, nearly error-free transmission was achieved at the expense of halving the average throughput. Compared to the uncoded system, the turbo-coded system was capable of achieving a higher throughput in the low SNR region at the expense of a higher complexity. The study of the relationship between the uncoded BER and the corresponding coded BER showed that adaptive modems obtain higher coding gains than that of fixed modems.
This was due to the fact that the adaptive modem avoids burst errors even in deep channel fades by reducing the number of bits per modulated symbol, eventually to zero.

\section{SUMMARY AND CONCLUSION}

We characterized the performance of various constant-power, adaptive-modulation schemes using the globally optimum switching levels based on the Lagrangian technique. In Section II-C1, the closed-form expressions were derived for the average BER, the average BPS throughput, and the MPS of the adaptive-modulation schemes, which were shown to be dependent on the mode-switching levels as well as on the average SNR. A set of relationships between the optimum mode-switching levels of a generic constant-power adaptive-modulation scheme was provided, where the relationships hold regardless of the operating channel scenario. The globally optimum switching levels were derived such that the average BER remains at the constant target average BER, while achieving the maximum possible BPS throughput for the given average SNR.

Then, in Section IV-A the performance of uncoded AQAM was characterized, when the underlying channel was a Nakagamifading channel. It was found that an adaptive scheme employing a $b_{k}$-BPS fixed-mode as the highest throughput constituent modulation mode achieved the highest possible BPS throughputs achievable by any link adaptation for the given SNR up to $b_{k-1}$ BPS. For example, a three-mode AQAM scheme employing No-Tx, 1-BPS BPSK, and 2-BPS QPSK modes attained the maximum possible average throughput up to 1 BPS. Hence, adding higher-throughput modes, such as 4-BPS 16-QAM to the three-mode AQAM scheme, which resulted in a four-mode AQAM scheme did not achieve a better BPS performance across the throughput range below 1 BPS. Instead, this four-mode AQAM scheme extended the maximal achievable average BPS throughput of the AQAM scheme to 2 BPS, while asymptotically achieving a throughput of 3 BPS as the average SNR increases. 
On the other hand, the relative SNR advantage of adaptive schemes increased in comparison to fixed-mode schemes as the target average BER became lower and decreased as the fading became less severe. More explicitly, less-severe fading corresponds to an increased Nakagami fading parameter $m$, to an increased number of diversity antennas, or to an increased number of multipath components encountered in wide-band fading channels. As the fading becomes less severe, the average BPS throughput curves of our adaptive square QAM schemes exhibit undulations owing to the absence of 3-, 5-, and 7-BPS square QAM modes.

The comparisons between fixed-mode MC-CDMA and adaptive OFDM (AOFDM) were made based on a number of different channel models in an effort to verify the applicability of our conclusions for different propagation scenarios. In Section IV-D, it was found that fixed-mode MC-CDMA might outperform adaptive OFDM, when the underlying channel provides sufficient diversity. However, this conclusion has to be verified by further research under more practicable conditions, since in practice MC-CDMA might suffer from MUI and AOFDM might suffer from imperfect channel-quality estimation and feedback delays.

Concatenated ST block-coded and turbo-convolutional-coded adaptive multicarrier systems were investigated in Section IV-E. The coded schemes reduced the required average SNR by about 6-7 dB at a throughput of 1 BPS, achieving near error-free transmission. It was also observed in Section IV-E that increasing the number of transmit antennas in adaptive schemes was not very effective, achieving less than 1-dB SNR gain, due to the fact that the transmit power per antenna had to be reduced in order to limit the total transmit power for the sake of fair comparison. In conclusion, adaptive modulation can be viewed as the lower complexity solution mitigating the wireless channel's quality fluctuations in comparison to multiple transmit- and receive-antenna-assisted ST-coded systems [4]. Additionally, it is important to note that while a conventional fixed-mode transceiver simply drops a call when the instantaneous channel quality is below a certain level, the adaptive transceivers drop their throughput, which is increased again during instances of high channel quality. This powerful technique has the potential for doubling the teletraffic capacity of the network when supported by intelligent networking procedures, as demonstrated by the results of [60].

\section{APPENDIX A BER OF QAM OVER AWGN CHANNEL}

The BER of fixed-mode QAM schemes communicating over AWGN channel is given as [2]

$$
p_{m}(\gamma)=\sum_{i} A_{i} Q\left(\sqrt{a_{i} \gamma}\right)
$$

where $Q(x)$ is the Gaussian $Q$-function defined as $Q(x) \triangleq$ $1 / \sqrt{2 \pi} \int_{x}^{\infty} e^{-t^{2} / 2} d t$ and $\left\{A_{i}, a_{i}\right\}$ is a set of modulation mode dependent constants. For the Gray-mapped square $m$-ary QAM modulation modes associated with $m=2,4,16,64$, and 256, the sets $\left\{A_{i}, a_{i}\right\}$ are given as [2], [61], [62]

$$
\begin{aligned}
& m=2, \text { BPSK }\{(1,2)\} \\
& m= \text { QPSK }\{(1,1)\} \\
& m=16,16-\text { QAM }\left\{\left(\frac{3}{4}, \frac{1^{2}}{5}\right),\left(\frac{2}{4}, \frac{3^{2}}{5}\right),\left(-\frac{1}{4}, \frac{5^{2}}{5}\right)\right\} \\
& m=64,64-\text { QAM }\left\{\left(\frac{7}{12}, \frac{1^{2}}{21}\right),\left(\frac{6}{12}, \frac{3^{2}}{21}\right),\left(-\frac{1}{12}, \frac{5^{2}}{21}\right),\right. \\
&\left.\left(\frac{1}{12}, \frac{9^{2}}{21}\right),\left(-\frac{1}{12}, \frac{13^{2}}{21}\right)\right\} \\
& m=256,256-\text { QAM }\left\{\left(\frac{15}{32}, \frac{1^{2}}{85}\right),\left(\frac{14}{32}, \frac{3^{2}}{85}\right),\left(\frac{5}{32}, \frac{5^{2}}{85}\right),\right. \\
&\left(-\frac{6}{32}, \frac{7^{2}}{85}\right),\left(-\frac{7}{32}, \frac{9^{2}}{85}\right), \\
&\left(\frac{6}{32}, \frac{11^{2}}{85}\right),\left(\frac{9}{32}, \frac{13^{2}}{85}\right),\left(\frac{8}{32}, \frac{15^{2}}{85}\right), \\
&\left(-\frac{7}{32}, \frac{17^{2}}{85}\right),\left(-\frac{6}{32}, \frac{19^{2}}{85}\right), \\
&\left(-\frac{1}{32}, \frac{21^{2}}{85}\right),\left(\frac{2}{32}, \frac{23^{2}}{85}\right),\left(\frac{3}{32}, \frac{25^{2}}{85}\right), \\
&\left.\left(-\frac{2}{32}, \frac{27^{2}}{85}\right),\left(-\frac{1}{32}, \frac{29^{2}}{85}\right)\right\} .
\end{aligned}
$$

\section{APPENDIX B}

\section{MODE-SPECIFIC AVERAGE BER OF AN ADAPTIVE MODULATION SCHEME}

A closed-form solution for the "mode-specific average BER" of a maximal ratio combining (MRC) receiver using $D$ th order antenna diversity for transmission over independent Rayleigh channels is derived, where the "mode-specific average BER" refers to the BER of the adaptive-modulation scheme, while activating one of its specific constituent modem modes. The pdf $f_{\bar{\gamma}}(\gamma)$ of the channel SNR $\gamma$ is given as [30, eq. (14-4-13)]

$$
f_{\bar{\gamma}}(\gamma)=\frac{1}{(D-1) ! \bar{\gamma}^{D}} \gamma^{D-1} e^{-\gamma / \bar{\gamma}} \gamma \geq 0
$$

where $\bar{\gamma}$ is the average channel SNR. Since the pdf of the instantaneous channel SNR $\gamma$ when communicating over a Nakagami-fading channel is given as

$$
f_{\bar{\gamma}}(\gamma)=\left(\frac{m}{\bar{\gamma}}\right)^{m} \frac{\gamma^{m-1}}{\Gamma(m)} e^{-m \gamma / \bar{\gamma}}, \gamma \geq 0
$$

the following results can also be applied to a Nakagami-fading channel with a simple change of variable given as $D=m$ and $\bar{\gamma}=\bar{\gamma} / m$.

The mode-specific average BER is defined as

$$
\begin{aligned}
& P_{r}(\alpha, \beta ; \bar{\gamma}, D, a) \\
\triangleq & \int_{\alpha}^{\beta} Q(\sqrt{a \gamma}) f_{\bar{\gamma}}(\gamma) d \gamma \quad(50) \\
= & \int_{\alpha}^{\beta} Q(\sqrt{a \gamma}) \frac{1}{(D-1) ! \bar{\gamma}^{D}} \gamma^{D-1} e^{-\gamma / \bar{\gamma}} d \gamma
\end{aligned}
$$


where $Q(x) \triangleq 1 / \sqrt{2 \pi} \int_{x}^{\infty} e^{-t^{2} / 2} d t$. Applying integration-by-part or $\int u d v=u v-\int v d u$ and noting that

$$
\begin{aligned}
u & =Q(\sqrt{a \gamma}) \\
d u & =-\frac{\sqrt{a}}{2 \sqrt{2 \pi \gamma}} e^{-a \gamma / 2} d \gamma \\
d v & =\frac{1}{(D-1) ! \bar{\gamma}^{D}} \gamma^{D-1} e^{-\gamma / \bar{\gamma}} d \gamma \\
v & =-e^{-\gamma / \bar{\gamma}} \sum_{d=0}^{D-1}\left(\frac{\gamma}{\bar{\gamma}}\right)^{d} \frac{1}{d !}
\end{aligned}
$$

(50) becomes

$$
\begin{array}{r}
P_{r}(\alpha, \beta ; \bar{\gamma}, D, a)=\left[e^{-\gamma / \bar{\gamma}} Q(\sqrt{a \gamma}) \sum_{d=0}^{D-1}\left(\frac{\gamma}{\bar{\gamma}}\right)^{d} \frac{1}{d !}\right]_{\beta}^{\alpha} \\
-\sum_{d=0}^{D-1} I_{d}(\alpha, \beta)
\end{array}
$$

where

$$
I_{d}(\alpha, \beta)=\int_{\alpha}^{\beta} \frac{\sqrt{a}}{2 \sqrt{2 \pi}} \frac{1}{d !}\left(\frac{\gamma}{\bar{\gamma}}\right)^{d} \frac{1}{\sqrt{\gamma}} e^{-a \gamma /\left(2 \mu^{2}\right)} d \gamma
$$

and $\mu=\sqrt{a \bar{\gamma} /(a \bar{\gamma}+2)}$. Let us consider $I_{d}$ for the case of $d=0$

$$
I_{0}(\alpha, \beta)=\int_{\alpha}^{\beta} \frac{\sqrt{a}}{2 \sqrt{2 \pi}} \frac{1}{\sqrt{\gamma}} e^{-a \gamma /\left(2 \mu^{2}\right)} d \gamma
$$

Upon introducing the variable $t^{2}=a \gamma / \mu^{2}$ and exploiting that $d \gamma=2 \mu \sqrt{\gamma / a} d t$, we have

$$
I_{0}(\alpha, \beta)=\left[\mu Q\left(\frac{\sqrt{a \gamma}}{\mu}\right)\right]_{\beta}^{\alpha} .
$$

Applying integration-by-part once to (53) yields

$$
\begin{array}{r}
I_{d}(\alpha, \beta)=\left[\frac{\mu^{2}}{\sqrt{2 a \pi}} \frac{1}{d !}\left(\frac{\gamma}{\bar{\gamma}}\right)^{d} \frac{1}{\sqrt{\gamma}} e^{-a \gamma /\left(2 \mu^{2}\right)}\right]_{\beta}^{\alpha} \\
+\frac{2 d-1}{a \bar{\gamma} d} \mu^{2} I_{d-1}
\end{array}
$$

which is a recursive form with the initial value given in (55). For this recursive form of (56), a nonrecursive form of $I_{d}$ can be expressed as

$$
\begin{aligned}
& I_{d}(\alpha, \beta) \\
& =\left[\frac{\mu^{2}}{\sqrt{2 a \pi}} \frac{\Gamma\left(d+\frac{1}{2}\right)}{\bar{\gamma}^{d} \Gamma(d+1)} \sum_{i=1}^{d}\left(\frac{2 \mu^{2}}{a}\right)^{d-i} \frac{\gamma^{i-1 / 2}}{\Gamma\left(i+\frac{1}{2}\right)} e^{-a \gamma /\left(2 \mu^{2}\right)}\right]_{\beta}^{\alpha} \\
& +\left[\left(\frac{2 \mu^{2}}{a \bar{\gamma}}\right)^{d} \frac{1}{\sqrt{\pi}} \frac{\Gamma\left(d+\frac{1}{2}\right)}{\Gamma(d+1)} \mu Q\left(\frac{\sqrt{a \gamma}}{\mu}\right)\right]_{\beta}^{\alpha} .
\end{aligned}
$$

By substituting $I_{d}(\alpha, \beta)$ of (57) into (52), the regional BER $P_{r}(\bar{\gamma} ; a, \alpha, \beta)$ can be represented in a closed form.

\section{APPENDIX C}

\section{RELATIONSHIP BETWEEN OPTIMUM SWITCHING LEVELS}

The optimum set of switching levels should satisfy

$$
\frac{\partial \Lambda}{\partial \mathbf{s}}=\frac{\partial}{\partial \mathbf{s}}\left(B(\bar{\gamma} ; \mathbf{s})+\lambda\left\{P_{R}(\bar{\gamma} ; \mathbf{s})-P_{t h} B(\bar{\gamma} ; \mathbf{s})\right\}\right)=0
$$

where $\Lambda(\mathbf{s} ; \bar{\gamma}), P_{R}(\bar{\gamma} ; \mathbf{s})$ and $B(\bar{\gamma} ; \mathbf{s})$ were given in (31), (28), and (8), respectively. The following results are helpful in evaluating the partial differentiations in (58):

$$
\begin{aligned}
\frac{\partial}{\partial s_{k}} P_{k-1} & =\frac{\partial}{\partial s_{k}} \int_{s_{k-1}}^{s_{k}} p_{m_{k-1}}(\gamma) f(\gamma) d \gamma \\
& =p_{m_{k-1}}\left(s_{k}\right) f\left(s_{k}\right) \\
\frac{\partial}{\partial s_{k}} P_{k} & =\frac{\partial}{\partial s_{k}} \int_{s_{k}}^{s_{k+1}} p_{m_{k}}(\gamma) f(\gamma) d \gamma \\
& =-p_{m_{k}}\left(s_{k}\right) f\left(s_{k}\right) \\
\frac{\partial}{\partial s_{k}} F_{c}\left(s_{k}\right) & =\frac{\partial}{\partial s_{k}} \int_{s_{k}}^{\infty} f(\gamma) d \gamma=-f\left(s_{k}\right) .
\end{aligned}
$$

Using (59) and (60), the partial differentiation of $P_{R}$, defined in (28) with respect to $s_{k}$, can be written as

$$
\frac{\partial P_{R}}{\partial s_{k}}=b_{k-1} p_{m_{k-1}}\left(s_{k}\right) f\left(s_{k}\right)-b_{k} p_{m_{k}}\left(s_{k}\right) f\left(s_{k}\right)
$$

where $b_{k}$ is the BPS throughput of an $m_{k}$-ary modem. Since the average throughput is given by $B=\sum_{k=0}^{K-1} c_{k} F_{c}\left(s_{k}\right)$ in (8), the partial differentiation of $B$ with respect to $s_{k}$ can be written using (61)

$$
\frac{\partial B}{\partial s_{k}}=-c_{k} f\left(s_{k}\right)
$$

where $c_{k}$ was defined as $c_{k} \triangleq b_{k}-b_{k-1}$ in Section II-A. Hence, (58) can be evaluated as

$$
\begin{array}{r}
{\left[-c_{k}\left(1-\lambda P_{t h}\right)+\lambda\left\{b_{k-1} p_{m_{k-1}}\left(s_{k}\right)-b_{k} p_{m_{k}}\left(s_{k}\right)\right\}\right] f\left(s_{k}\right)=0} \\
\text { for } k=1,2, \ldots, K-1 .
\end{array}
$$

A trivial solution of (64) is $f\left(s_{k}\right)=0$. Certainly, $\left\{s_{k}=\infty, k=1,2, \ldots, K-1\right\}$ satisfies this condition. Again, the lowest throughput modulation mode is "No-Tx" in our model, which corresponds to no transmission. When the pdf of $\gamma$ satisfies $f(0)=0,\left\{s_{k}=0, k=1,2, \ldots, K-1\right\}$ can also be a solution, which corresponds to the fixed-mode $m_{K-1}$-ary modem. The corresponding avalanche SNR $\bar{\gamma}_{\alpha}$, defined as the SNR beyond which only the highest throughput AQAM mode is used, can be obtained by substituting $\left\{s_{k}=0, k=1,2, \ldots, K-1\right\}$ into (34), which satisfies

$$
p_{m_{K-1}}\left(\bar{\gamma}_{\alpha}\right)-P_{t h}=0 .
$$


When $f\left(s_{k}\right) \neq 0$, (64) can be simplified upon dividing both sides by $f\left(s_{k}\right)$, yielding

$$
\begin{gathered}
-c_{k}\left(1-\lambda P_{t h}\right)+\lambda\left\{b_{k-1} p_{m_{k-1}}\left(s_{k}\right)-b_{k} p_{m_{k}}\left(s_{k}\right)\right\}=0 \\
\text { for } k=1,2, \ldots, K-1 .
\end{gathered}
$$

Rearranging (66) for $k=1$ and assuming $c_{1} \neq 0$, we have

$$
1-\lambda P_{t h}=\frac{\lambda}{c_{1}}\left\{b_{0} p_{m_{0}}\left(s_{1}\right)-b_{1} p_{m_{1}}\left(s_{1}\right)\right\} .
$$

Substituting (67) into (66) and assuming $c_{k} \neq 0$ for $k \neq 0$, we have

$$
\begin{aligned}
\frac{\lambda}{c_{k}}\left\{b_{k-1} p_{m_{k-1}}\left(s_{k}\right)\right. & \left.-b_{k} p_{m_{k}}\left(s_{k}\right)\right\} \\
& =\frac{\lambda}{c_{1}}\left\{b_{0} p_{m_{0}}\left(s_{1}\right)-b_{1} p_{m_{1}}\left(s_{1}\right)\right\} .
\end{aligned}
$$

In this context, we note that the Lagrangian multiplier $\lambda$ is not zero, since the substitution of $\lambda=0$ in (66) leads to $-c_{k}=$ 0 , which is not true. Hence, we can eliminate the Lagrangian multiplier upon dividing both sides of (68) by $\lambda$. Then we have

$$
y_{k}\left(s_{k}\right)=y_{1}\left(s_{1}\right) \text { for } k=2,3, \ldots K-1
$$

where the function $y_{k}\left(s_{k}\right)$ is defined as

$$
\begin{array}{r}
y_{k}\left(s_{k}\right) \triangleq \frac{1}{c_{k}}\left\{b_{k} p_{m_{k}}\left(s_{k}\right)-b_{k-1} p_{m_{k-1}}\left(s_{k}\right)\right\}, \\
k=2,3, \ldots K-1
\end{array}
$$

which does not contain the Lagrangian multiplier $\lambda$ and, hence, it will be referred to as the "Lagrangian-free function."

Let us now investigate some properties of the Lagrangian-free function $y_{k}\left(s_{k}\right)$ given in (70). Considering that $b_{k}>b_{k-1}$ and $p_{m_{k}}\left(s_{k}\right)>p_{m_{k-1}}\left(s_{k}\right)$, it is readily shown that the value of $y_{k}\left(s_{k}\right)$ is always positive. When $s_{k}=0, y_{k}\left(s_{k}\right)$ becomes

$$
\begin{aligned}
y_{k}(0) & \triangleq \frac{1}{c_{k}}\left\{b_{k} p_{m_{k}}(0)-b_{k-1} p_{m_{k-1}}(0)\right\} \\
& =\frac{1}{c_{k}}\left\{\frac{b_{k}}{2}-\frac{b_{k-1}}{2}\right\}=\frac{1}{2} .
\end{aligned}
$$

Note also that $s_{k}=0$ is a sufficient but not necessary condition for satisfying $y_{k}\left(s_{k}\right)=1 / 2$. We also note that we have $\lim _{s_{k} \rightarrow \infty} y_{k}\left(s_{k}\right)=0$. Since $0<y_{1}\left(s_{1}\right)=p_{m_{1}}\left(s_{1}\right)<1 / 2$ holds, we can conclude that there is at least one solution for $s_{k}$, satisfying (69) for the given $s_{1}$. In general, $y_{k}\left(s_{k}\right)$ may not be monotonic, although $y_{1}\left(s_{1}\right)$ is. When $y_{k}\left(s_{k}\right)$ is not monotonic in the range of $y_{k}\left(s_{k}\right)<1 / 2$, we have a set of $N=2 i+1$ number of roots $s_{k, n} \mid n=1,2, \ldots, N$ for (69) where $i$ is a positive integer. By considering the sign of $\partial \Lambda / \partial s_{k}$, it can be readily shown that $\Lambda$ reaches its local maxima at $s_{k, n}$, where $n$ is an odd integer. In order to arrive at the global maximum of $\Lambda$, all of these odd-numbered roots $s_{k}$ should be considered. However, our extensive numerical results have shown that a multiple-root scenario occurs only at high average SNRs, namely close to the avalanche SNR of $\bar{\gamma}_{a}$ when 64 QAM and 256 QAM are employed as the constituent modulation modes. We found that choosing the highest root always resulted in an insignificant performance loss in terms of the average throughput. By considering that $y_{1}\left(s_{1}\right)$ is a monotonically decreasing function and that $y_{k}\left(s_{k}\right)$ also decreases near the odd-indexed roots, since we have $y_{k}\left(s_{k, n}-\delta\right)>y_{1}\left(s_{1}\right)$ and $y_{k}\left(s_{k, n}+\delta\right)<y_{1}\left(s_{1}\right)$ around an odd-indexed root $s_{k, n}$, it can be concluded that

$$
\frac{d s_{k}}{d s_{1}}>0 \text { when } y_{k}\left(s_{k}\right)=y_{1}\left(s_{1}\right) .
$$

Equation (72) states that the $k$ th optimum switching level $s_{k}$ always increases, whenever the lowest optimum switching level, namely $s_{1}$, increases. Again, our extensive numerical evaluations have confirmed this argument.

\section{APPENDIX D \\ EXTREME VALUES OF $Y\left(\bar{\gamma} ; \mathbf{s}\left(s_{1}\right)\right)$}

In order to find the minima and the maxima of $Y\left(\bar{\gamma} ; \mathbf{s}\left(s_{1}\right)\right)$ defined in (41), we have to evaluate the derivative of $Y\left(\bar{\gamma} ; \mathbf{s}\left(s_{1}\right)\right)$ with respect to $s_{1}$, which can be expressed as

$$
\begin{aligned}
\frac{d Y}{d s_{1}} & =\sum_{k=1}^{K-1} \frac{\partial Y}{\partial s_{k}} \frac{d s_{k}}{d s_{1}} \\
& =\sum_{k=1}^{K-1} \frac{\partial}{\partial s_{k}}\left\{P_{R}-P_{t h} B\right\} \frac{d s_{k}}{d s_{1}} .
\end{aligned}
$$

Upon substituting (59) through (63) into (74) we have

$$
\begin{aligned}
& \frac{d Y}{d s_{1}} \\
= & \sum_{k=1}^{K-1}\left\{b_{k-1} p_{m_{k-1}}\left(s_{k}\right)-b_{k} p_{m_{k}}\left(s_{k}\right)+P_{t h} c_{k}\right\} f\left(s_{k}\right) \frac{d s_{k}}{d s_{1}} \\
= & \sum_{k=1}^{K-1}\left[\frac{c_{k}}{c_{1}}\left\{b_{0} p_{m_{0}}\left(s_{1}\right)-b_{1} p_{m_{1}}\left(s_{1}\right)\right\}+P_{t h} c_{k}\right] f\left(s_{k}\right) \frac{d s_{k}}{d s_{1}} \\
= & \frac{1}{c_{1}}\left\{b_{0} p_{m_{0}}\left(s_{1}\right)-b_{1} p_{m_{1}}\left(s_{1}\right)+P_{t h}\right\} \sum_{k=1}^{K-1} c_{k} f\left(s_{k}\right) \frac{d s_{k}}{d s_{1}} .
\end{aligned}
$$

Considering $f\left(s_{k}\right) \geq 0$ and exploiting $d s_{k} / d s_{1}>0$ given in (72), we can conclude from (75) that $d Y / d s_{1}=0$ has roots when $f\left(s_{k}\right)=0$ for all $k$ or when $b_{1} p_{m_{1}}\left(s_{1}\right)-b_{0} p_{m_{0}}\left(s_{1}\right)=$ $P_{t h}$. The former condition corresponds to either $s_{i}=0$ for some pdf $f(\gamma)$ or to $s_{k}=\infty$ for all pdfs. By contrast, when the condition of $b_{1} p_{m_{1}}\left(s_{1}\right)-b_{0} p_{m_{0}}\left(s_{1}\right)=P_{t h}$ is met, $d Y / d s_{1}=0$ has a unique solution. Investigating the sign of the first derivative between these zeros and using the mini-max analysis [26], which will be described with the aid of (76) to (83), we can conclude that $Y\left(\bar{\gamma} ; s_{1}\right)$ has a global minimum of $Y_{\min }<0$ at $s_{1}=\zeta$ such that $b_{1} p_{m_{1}}(\zeta)-b_{0} p_{m_{0}}(\zeta)=P_{t h}$ and a maximum of $Y_{\max 1}$ at $s_{1}=0$, as well as another maximum value $Y_{\max 2}=0^{-}$at $s_{1}=\infty$.

Since $Y\left(\bar{\gamma} ; s_{1}\right)$ has a maximum value at $s_{1}=\infty$, let us find the corresponding maximum value. Let us first consider 
$\lim _{s_{1} \rightarrow \infty} P_{\text {avg }}(\bar{\gamma} ; \mathbf{s})$, where upon exploiting (11) and (28) we have

$$
\begin{aligned}
\lim _{s_{1} \rightarrow \infty} P_{\mathrm{avg}}(\bar{\gamma} ; \mathbf{s}) & =\frac{\lim _{s_{1} \rightarrow \infty} P_{R}}{\lim _{s_{1} \rightarrow \infty} B} \\
& =\frac{0}{0} .
\end{aligned}
$$

When applying l'Hopital's rule and using (59) through (63), we have

$$
\begin{aligned}
\frac{\lim _{s_{1} \rightarrow \infty} P_{R}}{\lim _{s_{1} \rightarrow \infty} B} & =\frac{\lim _{s_{1} \rightarrow \infty} \frac{d}{d s_{1}} P_{R}}{\lim _{s_{1} \rightarrow \infty} \frac{d}{d s_{1}} B} \\
& =\lim _{s_{1} \rightarrow \infty} \frac{1}{c_{1}} b_{1} p_{m_{1}}\left(s_{1}\right)-b_{0} p_{m_{0}}\left(s_{1}\right) \\
& =0^{+}
\end{aligned}
$$

implying that $P_{\text {avg }}\left(\bar{\gamma} ; s_{k}\right)$ approaches zero from positive values when $s_{1}$ tends to $\infty$. Since, according to (11), (28), and (41), the function $Y\left(\bar{\gamma} ; \mathbf{s}\left(s_{1}\right)\right)$ can be written as $B\left(P_{\text {avg }}-P_{t h}\right)$, we have

$$
\begin{aligned}
\lim _{s_{1} \rightarrow \infty} Y\left(\bar{\gamma} ; s_{1}\right) & =\lim _{s_{1} \rightarrow \infty} B\left(P_{\mathrm{avg}}-P_{t h}\right) \\
& =\lim _{s_{1} \rightarrow \infty} B\left(0^{+}-P_{t h}\right) \\
& =0^{-} .
\end{aligned}
$$

Hence, $Y\left(\bar{\gamma} ; \mathbf{s}\left(s_{1}\right)\right)$ asymptotically approaches zero from negative values, as $s_{1}$ tends to $\infty$. From the analysis of the minimum and the maxima, we can conclude that the constraint function $Y\left(\bar{\gamma} ; \mathbf{s}\left(s_{1}\right)\right)$ defined in (41) has a unique zero only if $Y\left(\bar{\gamma} ; 0^{+}\right)>0$ at a switching value of $0<s_{1}<\zeta$, where $\zeta$ satisfies $b_{1} p_{m_{1}}(\zeta)-b_{0} p_{m_{0}}(\zeta)=P_{t h}$. By contrast, when $Y\left(\bar{\gamma} ; 0^{+}\right)<0$, the optimum switching levels are all zero and the adaptive-modulation scheme always employs the highest order constituent modulation mode.

\section{APPENDIX E}

\section{BER OF 2-D RAKE ASSISTED QAM}

An ideal RAKE receiver [33] combines all the signal powers scattered over $N$ paths in an optimal manner so that the instantaneous SNR per symbol at the RAKE receiver's output can be maximized [30]. The noise at the RAKE receiver's output is known to be Gaussian [30]. The SNR $\gamma_{d}$ at the $d$ th ideal RAKE receiver's output in Fig. 13 is given as [30]

$$
\gamma_{d}=\sum_{n=1}^{N} \gamma_{d, n}
$$

where $\gamma_{d, n}=E / N_{o} \alpha_{d, n}^{2}$ and $\left\{\alpha_{d, n}\right\}$ are assumed to be normalized, such that $\sum_{n=1}^{N} \alpha_{d, n}^{2}$ becomes unity. Since we assumed that each multipath component has an independent Rayleigh distribution, the characteristic function of $\gamma_{d}$ can be represented as [30, p. 802]

$$
\psi_{\gamma_{d}}(j v)=\prod_{n=1}^{N} \frac{1}{1-j v \bar{\gamma}_{d, n}}
$$

where $\gamma_{d, n}=E / N_{o} E\left[\alpha_{d, n}^{2}\right]$. Let us assume furthermore that each of the $D$ diversity channels of Fig. 13 has the same MIP, although in practical systems each of the $D$ channels may have a different MIP. Under this assumption, $\bar{\gamma}_{d, n}$ in (85) can be written as $\bar{\gamma}_{n}$. The total SNR per symbol, $\gamma$, at the output of the combiner in front of the demodulator depicted in Fig. 13, is given as

$$
\gamma=\sum_{d=1}^{D} \gamma_{d}
$$

while the characteristic function of $\gamma$, under the assumption of independent identical diversity channels, can be formulated as

$$
\psi_{\gamma}(j v)=\prod_{n=1}^{N} \frac{1}{\left(1-j v \bar{\gamma}_{n}\right)^{D}}
$$

Applying the well-known technique of partial fraction expansion (PFE) [26], $\psi_{\gamma}(j v)$ can be expressed as

$$
\psi_{\gamma}(j v)=\sum_{d=1}^{D} \sum_{n=1}^{N} \Lambda_{d, n} \frac{1}{\left(1-j v \bar{\gamma}_{n}\right)^{d}}
$$

where the constant $\Lambda_{d, n}$ can be found by equating (87) and (88).

The pdf of $\gamma, f_{\bar{\gamma}}(\gamma)$ can be found by applying the inverse Fourier transform of $\psi_{\gamma}(j v)$ in (88), which is given, with the aid of [30, p. 781, Eq. (14-4-13)] by

$$
f(\gamma)=\sum_{d=1}^{D} \sum_{n=1}^{N} \Lambda_{d, n} \frac{1}{(d-1) ! \bar{\gamma}_{n}^{d}} \gamma^{d-1} e^{-\gamma / \bar{\gamma}_{n}}
$$

Since we now have the pdf $f_{\bar{\gamma}}(\gamma)$ of the channel SNR, let us calculate the average BER of $m$-ary square QAM employing Gray mapping. The average BER $P_{e}$ can be expressed as [2], [30]

$$
P_{e, k}=\int_{0}^{\infty} p_{m_{k}}(\gamma) f(\gamma) d \gamma
$$

where $p_{m_{k}}(\gamma)$ is the BER of $m$-ary square QAM employing Gray mapping for transmission over Gaussian channels [2]

$$
p_{m_{k}}(\gamma)=\sum_{i} A_{i} Q\left(\sqrt{a_{i} \gamma}\right)
$$

where $Q(x)$ is the Gaussian $Q$-function defined as $Q(x) \triangleq$ $1 / \sqrt{2 \pi} \int_{x}^{\infty} e^{-t^{2} / 2} d t$ and $\left\{A_{i}, a_{i}\right\}$ is a set of modulation mode dependent constants, which are given, for example, in [2] and [62]. The average BER of $m$-ary QAM in our scenario can be calculated by substituting $p_{m_{k}}(\gamma)$ of (91) and $f_{\bar{\gamma}}(\gamma)$ of (89) into (90)

$$
\begin{aligned}
P_{e, k}(\bar{\gamma}) & =\int_{0}^{\infty} \sum_{i} A_{i} Q\left(\sqrt{a_{i} \gamma}\right) f(\gamma) d \gamma \\
& =\sum_{i} A_{i} P_{e}\left(\bar{\gamma} ; a_{i}\right)
\end{aligned}
$$


where each constituent BER $P_{e}\left(\bar{\gamma} ; a_{i}\right)$ is defined as

$$
P_{e}\left(\bar{\gamma} ; a_{i}\right)=\int_{0}^{\infty} Q\left(\sqrt{a_{i} \gamma}\right) f(\gamma) d \gamma
$$

Using the similarity of $f_{\bar{\gamma}}(\gamma)$ in (89) and the pdf of the SNR of a $D$-element antenna-diversity assisted MRC system transmitting over flat Rayleigh channels [30, p. 781], the closed-form solution for the component BER $P_{e}\left(\bar{\gamma} ; a_{i}\right)$ can be expressed as

$$
\begin{aligned}
& P_{e}\left(\bar{\gamma} ; a_{i}\right) \\
= & \sum_{d=1}^{D} \sum_{n=1}^{N} \frac{1}{\sqrt{2 \pi}} \int_{0}^{\infty} \int_{\sqrt{2 \gamma}}^{\infty} e^{-x^{2} / 2} \Lambda_{d, n} \frac{\gamma^{d-1} e^{-\gamma / \bar{\gamma}_{n}}}{(d-1) ! \bar{\gamma}_{n}^{d}} d x d \gamma \\
= & \sum_{d=1}^{D} \sum_{n=1}^{N} \Lambda_{d, n}\left\{\frac{1-\mu_{n}}{2}\right\}^{d} \sum_{i=0}^{d-1}\left(\begin{array}{c}
d-1+i \\
i
\end{array}\right)\left\{\frac{1}{2}\left(1+\mu_{n}\right)\right\}^{i}
\end{aligned}
$$

where $\mu_{n} \triangleq \sqrt{a_{i} \bar{\gamma}_{n} /\left(2+a_{i} \bar{\gamma}_{n}\right)}$ and the average SNR per symbol is $\bar{\gamma}=D \sum_{n=1}^{N} \bar{\gamma}_{n}$. Substituting $P_{e}\left(\bar{\gamma} ; a_{i}\right)$ of (95) into (93), the average BER of an $m$-ary QAM RAKE receiver using antenna diversity can be expressed in a closed form.

\section{APPENDIX F \\ PERFORMANCE OF 2-D RAKE-ASSISTED AQAM}

In this section, we consider the characteristic parameters of the ideal 2-D RAKE-assisted AQAM scheme described in Section IV-C and Appendix E.

\section{Average Throughput}

The average throughput $B(\bar{\gamma}, \mathbf{s})$ expressed in terms of bits per symbol (BPS) according to (8) is given by

$$
B(\bar{\gamma}, \mathbf{s})=\sum_{k=0}^{K-1} c_{k} F_{c}(\gamma)
$$

where the complementary cdf of the instantaneous $\operatorname{SNR} \gamma F_{c}(\gamma)$ can be expressed with the aid of (89) as

$$
F_{c}(\gamma)=\sum_{d=1}^{D} \sum_{n=1}^{N} \Lambda_{d, n} e^{-\gamma / \bar{\gamma}_{n}} \sum_{k=0}^{d-1} \frac{\left(\frac{\gamma}{\bar{\gamma}_{n}}\right)^{k}}{\Gamma(k+1)}
$$

where $\Gamma(x)$ is the Gamma function [26].

\section{APPENDIX G \\ AVERAGE BER}

The mode-specific average BER $P_{k}$ was defined in (10) as

$$
P_{k} \triangleq \int_{s_{k}}^{s_{k+1}} p_{m_{k}}(\gamma) f(\gamma) d \gamma
$$

Upon substituting $p_{m_{k}}(\gamma)$ of (91) and $f(\gamma)$ of (89) into (98), we have

$$
\begin{aligned}
P_{k} & =\sum_{i} A_{i} \sum_{d=1}^{D} \sum_{n=1}^{N} \Lambda_{d, n} \int_{s_{k}}^{s_{k+1}} Q\left(\sqrt{a_{i} \gamma}\right) \frac{\gamma^{d-1} e^{-\gamma / \bar{\gamma}_{n}}}{(d-1) ! \bar{\gamma}_{n}^{d}} d \gamma \\
& =\sum_{i} A_{i} \sum_{d=1}^{D} \sum_{n=1}^{N} \Lambda_{d, n} P_{R}\left(s_{k}, s_{k+1}, \bar{\gamma}_{n}, d, a_{i}\right)
\end{aligned}
$$

A closed-form expression for $P_{R}\left(s_{k}, s_{k+1}, \bar{\gamma}_{n}, d, a_{i}\right)$ can be found by applying change-of-variables repeatedly, which can be expressed as

$$
\begin{aligned}
P_{R}=\left[-e^{-\gamma / \bar{\gamma}_{n}} Q\left(\sqrt{a_{i} \gamma}\right)\right. & \left.\sum_{j=0}^{d-1} \frac{\left(\frac{\gamma}{\bar{\gamma}_{n}}\right)^{j}}{\Gamma(j+1)}\right]_{s_{k}}^{s_{k+1}} \\
& +\left[\sum_{j=0}^{d-1} X_{j}\left(\gamma ; \bar{\gamma}_{n} a_{i}\right)\right]_{s_{k}}^{s_{k+1}}
\end{aligned}
$$

where $[g(\gamma)]_{s_{k}}^{s_{k+1}} \triangleq g\left(s_{k+1}\right)-g\left(s_{k}\right)$ and $X_{j}$ is given by

$$
\begin{aligned}
X_{j}\left(\gamma ; \bar{\gamma}_{n}, a_{i}\right)= & \frac{\mu_{n}^{2}}{\sqrt{2 a_{i} \pi}} \frac{\Gamma\left(j+\frac{1}{2}\right)}{\bar{\gamma}_{n}^{j} \Gamma(j+1)} \\
& \times \sum_{k=1}^{j}\left(\frac{2 \mu_{n}^{2}}{a_{i}}\right)^{j-k} \frac{\gamma^{k-1 / 2}}{\Gamma\left(k+\frac{1}{2}\right)} e^{-a_{i} \gamma /\left(2 \mu_{n}^{2}\right)} \\
& +\left(\frac{2 \mu_{n}^{2}}{a_{i} \bar{\gamma}_{n}}\right)^{j} \frac{1}{\sqrt{\pi}} \frac{\Gamma\left(j+\frac{1}{2}\right)}{\Gamma(j+1)} \mu_{n} Q\left(\frac{\sqrt{a_{i} \gamma}}{\mu_{n}}\right)
\end{aligned}
$$

where, again, $\mu_{n} \triangleq \sqrt{a_{i} \bar{\gamma}_{n} /\left(2+a_{i} \bar{\gamma}_{n}\right)}$ and $\Gamma(x)$ is the Gamma function. Then, the average BER $P_{\text {avg }}(\bar{\gamma}, \mathbf{s})$ of our adaptivemodulation scheme can be represented using (11).

\section{REFERENCES}

[1] R. Steele and L. Hanzo, Eds., Mobile Radio Communications, 2nd ed. New York: IEEE Press; Wiley, 1999.

[2] L. Hanzo, W. T. Webb, and T. Keller, Single-and Multi-Carrier Quadrature Amplitude Modulation. New York: IEEE Press; Wiley, 2000.

[3] J. F. Hayes, "Adaptive feedback communications," IEEE Trans. Commun. Technol., vol. COM-16, pp. 29-34, 1968.

[4] L. Hanzo, C. Wong, and M. Yee, Adaptive Wireless Transceivers. New York: Wiley; IEEE Press, 2002.

[5] J. K. Cavers, "Variable rate transmission for Rayleigh fading channels," IEEE Trans. Commun. Technol., vol. COM-20, pp. 15-22, Feb. 1972.

[6] W. T. Webb and R. Steele, "Variable rate QAM for mobile radio," IEEE Trans. Commun., vol. 43, pp. 2223-2230, July 1995.

[7] R. Steele and W. T. Webb, "Variable rate QAM for data transmission over Rayleigh fading channels," in Proc. Wireless'91, Calgary , AB, Canada, 1991, pp. 1-14.

[8] M. L. Moher and J. H. Lodge, "TCMP-A modulation and coding strategy for rician fading channels," IEEE J. Select. Areas Commun., vol. 7, pp. 1347-1355, Dec. 1989.

[9] J. K. Cavers, "An analysis of pilot symbol assisted modulation for Rayleigh fading channels," IEEE Trans. Veh. Technol., vol. 40, pp. 686-693, Nov. 1991.

[10] S. Sampei and T. Sunaga, "Rayleigh fading compensation for QAM in land mobile radio communications," IEEE Trans. Veh. Technol., vol. 42, pp. 137-147, May 1993. 
[11] S. Otsuki, S. Sampei, and N. Morinaga, "Square QAM adaptive modulation/TDMA/TDD systems using modulation level estimation with Walsh function," Electron. Lett., vol. 31, pp. 169-171, Feb. 1995.

[12] W. C. Y. Lee, "Estimate of channel capacity in Rayleigh fading environment," IEEE Trans. Veh. Technol., vol. 39, pp. 187-189, Aug. 1990.

[13] A. J. Goldsmith and P. P. Varaiya, "Capacity of fading channels with channel side information," IEEE Trans. Inform. Theory, vol. 43, pp. 1986-1992, Nov. 1997.

[14] M. S. Alouini and A. J. Goldsmith, "Capacity of Rayleigh fading channels under different adaptive transmission and diversity-combining technique," IEEE Trans. Veh. Technol., vol. 48, pp. 1165-1181, July 1999.

[15] A. J. Goldsmith and S. Chua, "Variable rate variable power MQAM for fading channels," IEEE Trans. Commun., vol. 45, pp. 1218-1230, Oct. 1997.

[16] J. M. Torrance and L. Hanzo, "Optimization of switching levels for adaptive modulation in a slow Rayleigh fading channel," Electron. Lett., vol. 32, pp. 1167-1169, 1996.

[17] W. H. Press, S. A. Teukolsky, W. T. Vetterling, and B. P. Flannery, Numerical Recipies in C. Cambridge, U.K.: Cambridge Univ. Press, 1992.

[18] B. J. Choi, M. Münster, L. L. Yang, and L. Hanzo, "Performance of Rake receiver assisted adaptive-modulation based CDMA over frequency selective slow Rayleigh fading channel," Electron. Lett., vol. 37, pp. 247-249, 2001

[19] J. Paris, M. del Carmen Aguayo-Torres, and J. Entrambasaguas, "Optimum discrete-power adaptive QAM scheme for Rayleigh fading channels," IEEE Commun. Lett., vol. 5, pp. 281-283, July 2001.

[20] S. T. Chung and A. Goldsmith, "Degrees of freedom in adaptive modulation: A unified view," IEEE Trans. Commun., vol. 49, pp. 1561-1571, Sept. 2001.

[21] C. H. Wong and L. Hanzo, "Upper-bound performance of a wideband burst-by-Burst adaptive modem," IEEE Trans. Commun., vol. 48, pp. 367-369, Mar. 2000.

[22] E. L. Kuan, C. H. Wong, and L. Hanzo, "Burst-by-burst adaptive jointdetection CDMA," Proc. IEEE Vehicular Technology Conf.'99 Fall, vol. 2, pp. 1628-1632, Sept. 1999.

[23] J. M. Torrance and L. Hanzo, "Upper bound performance of adaptive modulation in a slow Rayleigh fading channel," Electron. Lett., vol. 32, pp. 718-719, 1996

[24] M. Nakagami, "The $m$-distribution - A general formula of intensity distribution of rapid fading," in Statistical Methods in Radio Wave Propagation, W. C. Hoffman, Ed. New York: Pergamon, 1960, pp. 3-36.

[25] I. S. Gradshteyn and I. M. Ryzhik, Table of Integrals, Series and Products. New York: Academic, 1980.

[26] E. Kreyszig, Advanced Engineering Mathematics, 7th ed. New York: Wiley, 1993.

[27] J. Lu, K. B. Letaief, C. I. J. Chuang, and M. L. Lio, "M-PSK and M-QAM BER computation using signal-space concepts," IEEE Trans. Commun., vol. 47, no. 2, pp. 181-184, Feb. 1999

[28] B. J. Choi, "Multi Carrier Code Division Multiple Access," PhD, University of Southampton, Dept. Electron Computer Sci., Southampton, U.K., 2001.

[29] G. S. G. Beveridge and R. S. Schechter, Optimization: Theory and Practice. New York: McGraw-Hill, 1970.

[30] J. G. Proakis, Digital Communications, 3rd ed. New York: Mc-Graw Hill, 1995.

[31] W. Jakes Jr, Ed., Microwave Mobile Communications. New York: Wiley, 1974

[32] “COST 207: Digital Land Mobile Radio Communications," Office for Official Publications of the European Communities, Luxembourg, 1989.

[33] R. Price and E. Green Jr, "A communication technique for multipath channels," Proc. IRE, vol. 46, pp. 555-570, Mar. 1958.

[34] M. K. Simon and M. S. Alouini, Digital Communication Over Fading Channels: A Unified Approach to Performance Analysis. New York: Wiley, 2000

[35] C. Y. Wong, R. S. Cheng, K. B. Letaief, and R. D. Murch, "Multiuser OFDM with adaptive subcarrier, bit, and power allocation," IEEE J. Select. Areas Commun., vol. 17, pp. 1747-1758, Oct. 1999.

[36] T. Keller and L. Hanzo, "Adaptive multicarrier modulation: A convenient framework for time-frequency processing in wireless communications," Proc. IEEE, vol. 88, pp. 611-642, May 2000.

[37] J. Bingham, "Multicarrier modulation for data transmission: An idea whose time has come," IEEE Commun. Mag., pp. 5-14, May 1990

[38] N. Yee, J. P. Linnartz, and G. Fettweis, "Multicarrier CDMA in indoor wireless radio networks," in Proc. PIMRC'93 , 1993, pp. 109-113.
[39] K. Fazel and L. Papke, "On the performance of convolutionally-coded CDMA/OFDM for mobile communication system," in Proc. PIMRC'93, 1993, pp. 468-472.

[40] A. Klein, G. K. Kaleh, and P. W. Baier, "Zero forcing and minimum mean square error equalization for multiuser detection in code division multiple access channels," IEEE Trans.Veh. Technol., vol. 45, pp. 276-287, May 1996.

[41] L. Hanzo and B. Y. T. H. Liew, Turbo Coding, Turbo Equalization and Space-Time Coding. New York: Wiley, IEEE Press, 2002.

[42] B. Vucetic, "An adaptive coding scheme for time-varying channels," IEEE Trans. Commun., vol. 39, pp. 653-663, May , 1991.

[43] H. Imai and S. Hirakawa, "A new multi-level coding method using error correcting codes," IEEE Trans. Inform. Theory, vol. IT-23, pp. 371-377, May 1977.

[44] G. Ungerboeck, "Channel coding with multilevel/phase signals," IEEE Trans. Inform. Theory, vol. IT-28, pp. 55-67, Jan. 1982.

[45] S. M. Alamouti and S. Kallel, "Adaptive trellis-coded multiple-phasedshift keying Rayleigh fading channels," IEEE Trans. Commun., vol. 42 , pp. 2305-2341, June 1994.

[46] S. Chua and A. J. Goldsmith, "Adaptive coded modulation for fading channels," IEEE Trans. Commun., vol. 46, pp. 595-602, May 1998.

[47] K. J. Hole, H. Holm, and G. E. Oien, "Adaptive multidimensional coded modulation over flat fading channels," IEEE J. Select. Areas Commun., vol. 18, pp. 1153-1158, July 2000.

[48] S. X. Ng, C. H. Wong, and L. Hanzo, "Burst-by-Burst adaptive decision feedback equalized tcm, ttcm, bicm and bicm-id," in Proc. IEEE ICC'01, June 2001, pp. 3031-3035.

[49] T. Keller, T. Liew, and L. Hanzo, "Adaptive rate RRNS coded OFDM transmission for mobile communication channels," in Proc. Vehicular Technology Conf. 2000 Spring, Tokyo, Japan, May 15-18, 2000, pp. 230-234.

[50] T. Keller, T. H. Liew, and L. Hanzo, "Adaptive redundant residue number system coded multicarrier modulation," IEEE J. Select. Areas Commun., vol. 18, pp. 1292-2301, Nov. 2000.

[51] T. Liew, C. Wong, and L. Hanzo, "Block turbo coded burst-by-burst adaptive modems," in Proc. Microcoll'99, Budapest, Hungary, Mar. 1999 , pp. 59-62.

[52] C. Wong, T. Liew, and L. Hanzo, "Turbo coded burst by burst adaptive wideband modulation with blind modem mode detection," in Proc. ACTS Mobile Communications Summit, Sorrento, Italy, June 1999, pp. 303-308.

[53] M. Yee, T. Liew, and L. Hanzo, "Radial basis function decision feedback equalization assisted block turbo burst-by-burst adaptive modems," in Proc. Vehnicular Technology Conf.'99 Fall , Amsterdam, The Netherlands, Sept. 1999, pp. 1600-1604.

[54] V. Tarokh, N. Seshadri, and A. Calderbank, "Space-time codes for high data rate wireless communication: Performance criterion and code construction," IEEE Trans. Inform. Theory, vol. 44, pp. 744-765, Mar. 1998

[55] S. M. Alamouti, "A simple transmit diversity technique for wireless communications," IEEE J. Select. Areas Commun., vol. 16, pp. $1451-1458$, Oct. 1998.

[56] V. Tarokh, H. Jafarkhani, and A. R. Calderbank, "Space-time block coding for wireless communications: Performance results," IEEE $J$. Select. Areas Commun., vol. 17, pp. 451-460, Mar. 1999.

[57] C. Berrou and A. Glavieux, "Near optimum error correcting coding and decoding: Turbo-codes," IEEE Trans. Commun., vol. 44, pp. 1261-1271, Oct. 1996.

[58] T. Liew, J. Pliquett, B. Yeap, L.-L. Yang, and L. Hanzo, "Concatenated space time block codes and TCM, turbo TCM, convolutional as well as turbo codes," in Proc. IEEE GLOBECOM'00, San Francisco, CA, Nov.-Dec. 27-1, 2000

[59] B. J. Choi, E. L. Kuan, and L. Hanzo, "Crest-factor study of MC-CDMA and OFDM," in Proc. Vehicular Technology Conf.' 99 Fall, vol. 1, Amsterdam, The Netherlands, Sept. 19-22, 1999, pp. 233-237.

[60] J. Blogh and L. Hanzo, $3 G$ Systems and Intelligent Networking. New York: Wiley; IEEE Press, 2002.

[61] M. S. Alouini, X. Tand, and A. J. Goldsmith, "An adaptive modulation scheme for simultaneous voice and data transmission over fading channels," IEEE J. Select Areas Commun., vol. 17, pp. 837-850, May 1999.

[62] D. Yoon, K. Cho, and J. Lee, "Bit error probability of M-ary quadrature amplitude modulation," Proc. IEEE Vehicular Technology Conf. 2000 Fall, vol. 5, pp. 2422-2427, Sept. 2000. 


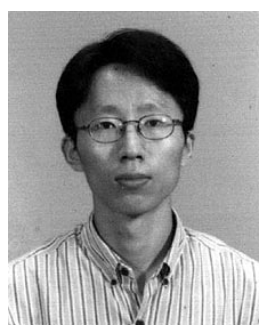

Byoungjo Choi received the B.Sc. and M.Sc. degrees in electrical engineering from KAIST, Korea, in 1990 and 1992, respectively. He received the $\mathrm{Ph} . \mathrm{D}$. degree in mobile communications from the University of Southampton, U.K.

He has been with LG Electronics, Korea, since January, 1992, where he was involved in developing the KoreaSat monitoring system, digital DBS transmission system, and W-CDMA based wireless local loop (WLL) system. He was a Postdoctoral Research Assistant at the University of Southampton, U.K., from September 2001 to August 2002. His current research interests include mobile communication systems design with empasis on adaptive modulation aided OFDM, MC-CDMA, and W-CDMA.

Dr. Choi is a recipient of the British Chevening Scholarship awarded by the British Council, U.K.

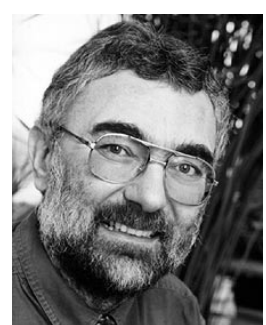

Lajos Hanzo (SM'92) received the Master's degree in electronics, in 1976 and the Ph.D. degree in telecommunications in 1983, both from the Technical University of Budapest, Hungary.

During his 27-year career in telecommunications, he has held various research and academic posts in Hungary, Germany, and the United Kingdom. Since 1986, he has been with the Department of Electronics and Computer Science, University of Southampton, Southampton, U.K., where he holds the Chair in telecommunications. Currently, he is managing an academic research team, working on a range of research projects in the field of wireless multimedia communications sponsored by industry, the Engineering and Physical Sciences Research Council (EPSRC) U.K., the European IST Programme, and the Mobile Virtual Centre of Excellence (V.C.E), U.K. He is an enthusiastic supporter of industrial and academic liaison and offers a range of industrial courses. He has coauthored 10 IEEE Press-Wiley books on mobile radio communications, published in excess of 400 research papers (http://www-mobile.ecs.soton.ac.uk).

Dr. Hanzo organized and chaired conference sessions, presented overview lectures, has been awarded a number of distinctions, and is also an IEEE Distinguished Lecturer. 